\title{
Design of Two-Step Random Access Procedure for URLLC Applications
}

\author{
Chih-Cheng Tseng ${ }^{1} \cdot$ Hwang-Cheng Wang ${ }^{2} \cdot$ Jieh-Ren Chang ${ }^{2} \cdot$ Ling-Han Wang $^{1}$. \\ Fang-Chang Kuo ${ }^{2}$ (D)
}

Accepted: 16 August 2021 / Published online: 8 September 2021

(c) The Author(s), under exclusive licence to Springer Science+Business Media, LLC, part of Springer Nature 2021

\begin{abstract}
The International Telecommunication Union has required that the control plane (C-plane) latency in the fifth generation (5G) ultra-reliable low-latency communication (URLLC) application scenarios should not exceed $20 \mathrm{~ms}$ and encouraged technical innovation to further reduce it to less than $10 \mathrm{~ms}$. However, the average C-plane latency in the fourth generation (4G) Long Term Evolution-Advanced (LTE-A) system is $80 \mathrm{~ms}$. Such a high latency is because of the execution of the contention-based random access procedure (RAP). In this paper, we simplify the conventional contention-based RAP from 4 to 2 steps. Furthermore, utilization of demodulation reference signal for representing the UE ID and reservation of preambles for URLLC users significantly reduces the proposed 2-step RAP latency. From the perspectives of fixing the number of URLLC users and fixing the number of preambles reserved for URLLC users, simulation results show the percentage of successes for the 2-step RAP is $83.81 \%$ and $71.83 \%$ higher than that of the 4-step RAP, respectively. Consequently, the $10 \mathrm{~ms}$ latency requirement of the 5G URLLC is achieved.
\end{abstract}

Keywords Control plane latency $\cdot$ Demodulation reference signal $\cdot$ Preamble $\cdot$ Random access procedure $\cdot$ URLLC

\section{Introduction}

In the fourth generation (4G) Long Term Evolution-Advanced (LTE-A) system, the uplink (UL) connection setup procedure includes three phases: Random Access Procedure (RAP), Authentication and Security (AS), and Radio Resource Management (RRM). These three phases are performed in the control plane (C-plane). RAP can be classified into non-contention-based and contention-based [1]. In the non-contention-based RAP, the base station directly assigns a preamble to a user equipment (UE). Then, the UE decides whether to perform RAP by itself. Because the preambles assigned to the UEs by the base station are different, collision of preambles at the base station will not occur in the non-contention-based

Fang-Chang Kuo

kfc@niu.edu.tw

1 Department of Electrical Engineering, National Ilan University, Taiwan, Republic of China

2 Department of Electronic Engineering, National Ilan University, Taiwan, Republic of China 
RAP. Conversely, if a UE wants to perform a contention-based RAP, it must arbitrarily select a preamble from the set with a limited number of preambles and send it to the base station. Since the preamble is simply an orthogonal code, the base station only knows which preamble is detected but cannot identify how many UEs send the detected preamble. If a detected preamble is sent by multiple UEs, those UEs that sent the same preamble will get the same uplink resource allocated by the base station. As a result, multiple Message 3 (Msg 3) messages collide at the base station. In this case, all the corresponding UEs must re-initiate the contention-based RAP, resulting in longer latency. This explains why the C-plane latency of the contention-based RAP is so high in the 4G LTE-A system.

In response to hardware innovation and technology development, 3GPP Release 15 has defined various performance indexes for the fifth generation $(5 \mathrm{G})$ application scenarios. Compared with the performance of $4 \mathrm{G}$ LTE-A, these indexes can be very different. For example, the average C-plane latency of 4G LTE-A is $80 \mathrm{~ms}$. However, the C-plane latency for ultra-reliable low-latency communication (URLLC) application scenarios is expected to be below $10 \mathrm{~ms}$. In Release 15 [2], the contention-based RAP in 5G still maintains the same four steps in the 4G LTE-A system. Thus, there are also 64 preambles available in the base station. Obviously, with such a limited number of preambles, as the number of 5 G UEs increases, the congestion of UEs in the RAP will increase tremendously. Once the congestion increases, the latency of RAP will increase accordingly, making it challenging to meet the $10 \mathrm{~ms}$ C-plane latency requirement.

The contributions of this paper are:

1. The architecture of the 4-step RAP is simplified to 2-step RAP to reduce the C-plane latency.

2. A certain number of preambles are reserved and allocated to URLLC users exclusively to reduce the collision of the preambles selected by URLLC users. Once the number of users contending for the preambles is diminished, the percentage of RAP success is improved. In this paper, when the latency to complete RAP is below $10 \mathrm{~ms}$, the RAP is considered successful.

3. The DMRS is used to represent the UE ID to take advantage of the orthogonality property.

The rest of this paper is structured as follows. Section 2 provides a brief review of the related technical background. Section 3 explains the operation of the existing 4-step RAP. The detailed design and operation of the proposed 2-step RAP are presented in Sect. 4. Simulation results and performance comparison are given in Sect. 5. Section 6 concludes this paper.

\section{Preliminaries}

\subsection{Physical Random Access Channel (PRACH)}

The PRACH occupies six consecutive resource blocks (RBs) in the frequency domain for a total of $1.08 \mathrm{MHz}$. In 4-step RAP, the transmission of preamble must be carried out in the PRACH. As can be seen in Fig. 1, the preamble is composed of three parts: Cyclic Prefix (CP), Sequence, and Guard time (GT). In addition, the subcarrier spacing of PRACH is $1.25 \mathrm{kHz}$, while the subcarrier spacing of a UL channel is $15 \mathrm{kHz}$. Therefore, the total 


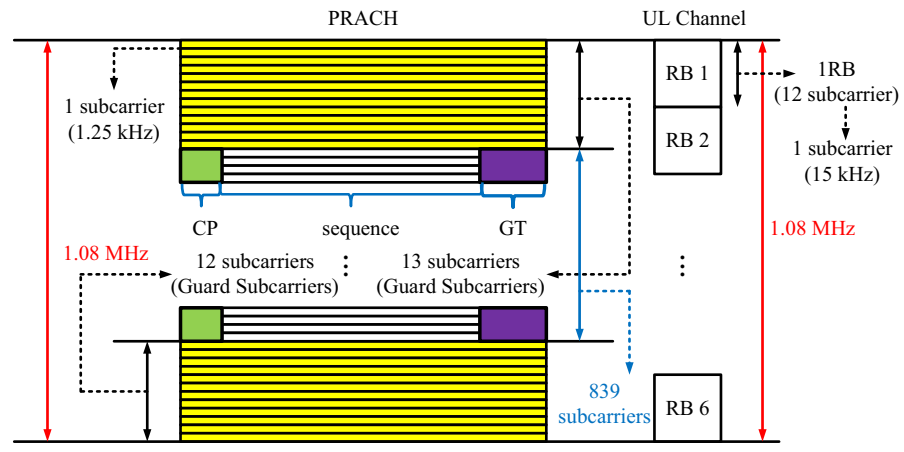

Fig. 1 Comparison of PRACH and UL channel

number of subcarriers in PRACH and UL channel are 864 and 72, respectively. At the top and bottom ends of the PRACH, 13 and 12 subcarriers are reserved respectively for guard subcarriers. Hence, only 839 subcarriers are used to transmit the preamble.

\subsection{System Information Block 2 (SIB2)}

System Information (SI) can be divided into Master Information Block (MIB) and System Information Block (SIB). In response to different applications and requirements, there are 13 different SIBs. However, to perform RAP, a UE needs to listen to the SIB2 broadcast from the base station. SIB2 contains the following important system parameters for RAP:

1. rootSequenceIndex As shown in Table 1, this parameter indicates the physical root sequence number $u$ for generating the preamble in Msg 1 of the 4-step RAP.

2. prach-ConfigIndex As shown in Table 2, this parameter indicates the preamble format, the system frame number(s), and the subframe number(s) that can be used to transmit the generated preamble. With Table 2, this parameter provides the UE the following three pieces of information: (i) The preamble format. There are four possible preamble formats, numbered 0-3. (ii) The system frames to transmit the preamble. The preamble can be sent on either an even-numbered system frame or any system frame. (iii) The subframe to transmit the preamble, which is also regarded as the random access opportunity (RAO). As indicated in Table 2, the preamble can be sent on one of the 10 subframes of a system frame if prach-ConfigIndex equals 14. Figure 2 illustrates the subframes that can be used to transmit a preamble for the six possible values of prach-ConfigIndex. For example, if the value of prach-ConfigIndex broadcast by the base station in SIB2 is 12, the preamble format 0 will be used, and any subframe numbered $0,2,4,6$, and 8 can be used to transmit the preamble.

3. prach-FreqOffset This parameter indicates the frequency in which a UE transmits the preamble. The prach-FreqOffset in FDD mode defined in [3] is the first physical resource block (PRB) allocated to the PRACH opportunity considered for preamble formats 0 , 1,2 , and 3, and is defined as $n_{\mathrm{PRB}}^{\mathrm{RA}}=n_{\mathrm{PRB} \text { offset }}^{\mathrm{RA}} . n_{\mathrm{PRB}}^{\mathrm{RA}}$ is the first PRB occupied by PRACH resource considered. $n_{\mathrm{PRB} \text { offset }}^{\mathrm{RA}}$ is the value of the parameter prach-FreqOffset expressed as the PRB number configured by higher layers and satisfies $0 \leq n_{\mathrm{PRB} \text { offset }}^{\mathrm{RA}} \leq N_{\mathrm{RB}}^{\mathrm{UL}}-6$. In practice, $n_{\mathrm{PRB} \text { offset }}^{\mathrm{RA}}$ is the first PRB available for PRACH. $N_{\mathrm{RB}}^{\mathrm{UL}}$ is the uplink bandwidth configuration, expressed in multiples of $N_{\mathrm{sc}}^{\mathrm{RB}} \cdot N_{\mathrm{sc}}^{\mathrm{RB}}$ is the RB size in the frequency 
Table 1 Root Zadoff-Chu sequence order for preamble formats 0-3 (FDD) [3]

\begin{tabular}{ll}
\hline Logical root & Physical root sequence number $u$ (in increasing order of the corresponding logical \\
sequence & sequence number)
\end{tabular}

number

\begin{tabular}{ll}
\hline $0-23$ & $129,710,140,699,120,719,210,629,168,671,84,755,105,734,93,746,70,769,60$, \\
& $779,2,837,1,838$ \\
$24-29$ & $56,783,112,727,148,691$ \\
$30-35$ & $80,759,42,797,40,799$ \\
$36-41$ & $35,804,73,766,146,693$ \\
$42-51$ & $31,808,28,811,30,809,27,812,29,810$ \\
$52-63$ & $24,815,48,791,68,771,74,765,178,661,136,703$ \\
$64-75$ & $86,753,78,761,43,796,39,800,20,819,21,818$ \\
$76-89$ & $95,744,202,637,190,649,181,658,137,702,125,714,151,688$ \\
$90-115$ & $217,622,128,711,142,697,122,717,203,636,118,721,110,729,89,750,103,736$, \\
& $61,778,55,784,15,824,14,825$ \\
$116-135$ & $12,827,23,816,34,805,37,802,46,793,207,632,179,660,145,694,130,709,223$, \\
& 616 \\
$136-167$ & $228,611,227,612,132,707,133,706,143,696,135,704,161,678,201,638,173,666$, \\
& $106,733,83,756,91,748,66,773,53,786,10,829,9,830$ \\
$168-203$ & $7,832,8,831,16,823,47,792,64,775,57,782,104,735,101,738,108,731,208,631$, \\
& $184,655,197,642,191,648,121,718,141,698,149,690,216,623,218,621$ \\
$204-263$ & $152,687,144,695,134,705,138,701,199,640,162,677,176,663,119,720,158,681$, \\
& $164,675,174,665,171,668,170,669,87,752,169,670,88,751,107,732,81,758$, \\
& $82,757,100,739,98,741,71,768,59,780,65,774,50,789,49,790,26,813,17,822$, \\
& $13,826,6,833$
\end{tabular}

264-327 5, 834, 33, 806, 51, 788, 75, 764, 99, 740, 96, 743, 97, 742, 166, 673, 172, 667, 175, 664, $187,652,163,676,185,654,200,639,114,725,189,650,115,724,194,645,195$, $644,192,647,182,657,157,682,156,683,211,628,154,685,123,716,139,700$, $212,627,153,686,213,626,215,624,150,689$

328-383 225, 614, 224, 615, 221, 618, 220, 619, 127, 712, 147, 692, 124, 715, 193, 646, 205, 634, $206,633,116,723,160,679,186,653,167,672,79,760,85,754,77,762,92,747,58$, $781,62,777,69,770,54,785,36,803,32,807,25,814,18,821,11,828,4,835$

384-455 3, 836, 19, 820, 22, 817, 41, 798, 38, 801, 44, 795, 52, 787, 45, 794, 63, 776, 67, 772, 72, $767,76,763,94,745,102,737,90,749,109,730,165,674,111,728,209,630,204$, $635,117,722,188,651,159,680,198,641,113,726,183,656,180,659,177,662$, $196,643,155,684,214,625,126,713,131,708,219,620,222,617,226,613$

456-513

$230,609,232,607,262,577,252,587,418,421,416,423,413,426,411,428,376,463$, $395,444,283,556,285,554,379,460,390,449,363,476,384,455,388,451,386$, $453,361,478,387,452,360,479,310,529,354,485,328,511,315,524,337,502$, $349,490,335,504,324,515$

514-561 323, 516, 320, 519, 334, 505, 359, 480, 295, 544, 385, 454, 292, 547, 291, 548, 381, 458, $399,440,380,459,397,442,369,470,377,462,410,429,407,432,281,558,414$, $425,247,592,277,562,271,568,272,567,264,575,259,580$

$562-629$

$237,602,239,600,244,595,243,596,275,564,278,561,250,589,246,593,417,422$, $248,591,394,445,393,446,370,469,365,474,300,539,299,540,364,475,362$, $477,298,541,312,527,313,526,314,525,353,486,352,487,343,496,327,512$, $350,489,326,513,319,520,332,507,333,506,348,491,347,492,322,517$

$630-659$

$330,509,338,501,341,498,340,499,342,497,301,538,366,473,401,438,371,468$, $408,431,375,464,249,590,269,570,238,601,234,605$

660-707 257, 582, 273, 566, 255, 584, 254, 585, 245, 594, 251, 588, 412, 427, 372, 467, 282, 557, 403, 436, 396, 443, 392, 447, 391, 448, 382, 457, 389, 450, 294, 545, 297, 542, 311, $528,344,495,345,494,318,521,331,508,325,514,321,518$ 
Table 1 (continued)

\begin{tabular}{ll}
$\begin{array}{l}\text { Logical root } \\
\text { sequence } \\
\text { number }\end{array}$ & $\begin{array}{l}\text { Physical root sequence number } u \text { (in increasing order of the corresponding logical } \\
\text { sequence number) }\end{array}$ \\
\hline $708-729$ & $\begin{array}{l}346,493,339,500,351,488,306,533,289,550,400,439,378,461,374,465,415,424, \\
270,569,241,598\end{array}$ \\
$730-751$ & $231,608,260,579,268,571,276,563,409,430,398,441,290,549,304,535,308,531$, \\
& $358,481,316,523$ \\
$752-765$ & $293,546,288,551,284,555,368,471,253,586,256,583,263,576$ \\
$766-777$ & $242,597,274,565,402,437,383,456,357,482,329,510$ \\
$778-789$ & $317,522,307,532,286,553,287,552,266,573,261,578$ \\
$790-795$ & $236,603,303,536,356,483$ \\
$796-803$ & $355,484,405,434,404,435,406,433$ \\
$804-809$ & $235,604,267,572,302,537$ \\
$810-815$ & $309,530,265,574,233,606$ \\
$816-819$ & $367,472,296,543$ \\
$820-837$ & $336,503,305,534,373,466,280,559,279,560,419,420,240,599,258,581,229,610$ \\
\hline
\end{tabular}

domain, expressed as a multiple of subcarriers. For example, if $N_{\mathrm{RB}}^{\mathrm{UL}}=100$, the value of prach-FrequencyOffset will be selected between 0 and 94. If prach-FrequencyOff$s e t=55$, the $6 \mathrm{RBs}$ numbered from 55 to 60 are used to send preamble.

4. preambleTransMax This parameter specifies the number of times a UE can retransmit the preamble in the RAP. As mentioned in [4], the value of preambleTransMax can be 3 , $4,5,6,7,8,10,20,50,100$, and 200. When the number of preamble retransmissions is below preambleTransMax, RAP must be restarted, and the preambe_transmission_counter will be increased by 1 . Otherwise, the UE declares RAP failure.

5. numberOfRA-Preambles The number of preambles reserved for contention-based RAP. Its value is usually 54.

6. mac-ContentionResolutionTimer This parameter is the waiting time required for a UE to collect the Msg 4 transmitted by the base station after a UE transmits Msg 3. In this paper, if a UE does not receive Msg 4 within this time limit because multiple UEs collide over Msg 3, collision resolution is deemed to have failed. As mentioned in [4], the value of mac-ContentionResolutionTimer can be sf8, sf16, sf24, sf32, sf40, sf48, sf56, and sf64, where sf is the subframe time, which is $1 \mathrm{~ms}$.

\section{4-Step RAP}

As mentioned earlier, since the preamble in the non-contention-based RAP is assigned by the base station, there will be no collision during RAP. Hence, this paper only discusses the contention-based RAP. There are six opportunities for a UE to initiate contention-based RAP [1]: (i) a UE needs to perform initial access to evolved Node B (eNodeB) from RRC (Radio Resource Control)_IDLE state; (ii) a UE wants to re-establish RRC connection; (iii) a UE has uplink data to be transmitted but its uplink synchronization status shows that it is asynchronous; (iv) a UE has uplink data to transmit but does not have PUCCH resources to transmit the uplink scheduling request (SR); (v) eNodeB has to perform downlink 


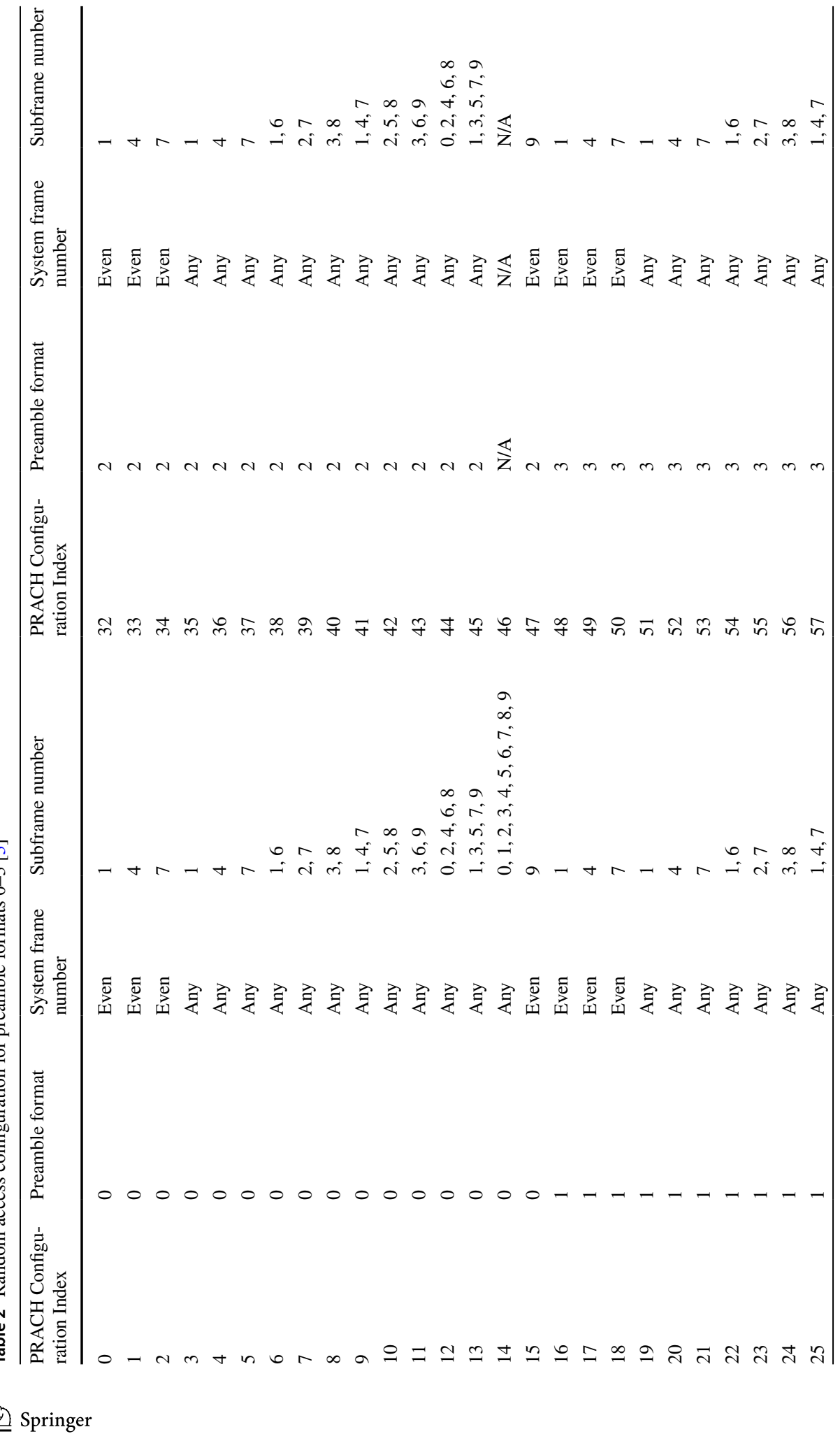




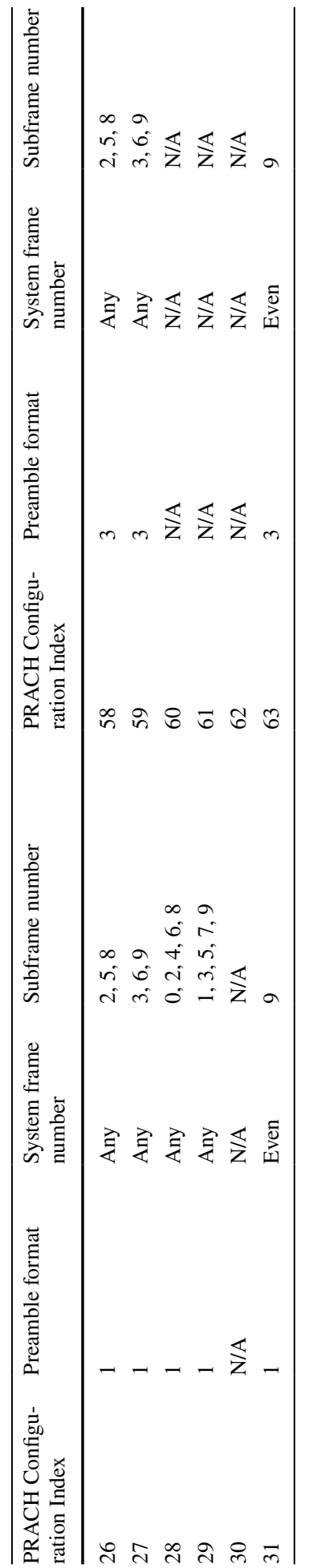




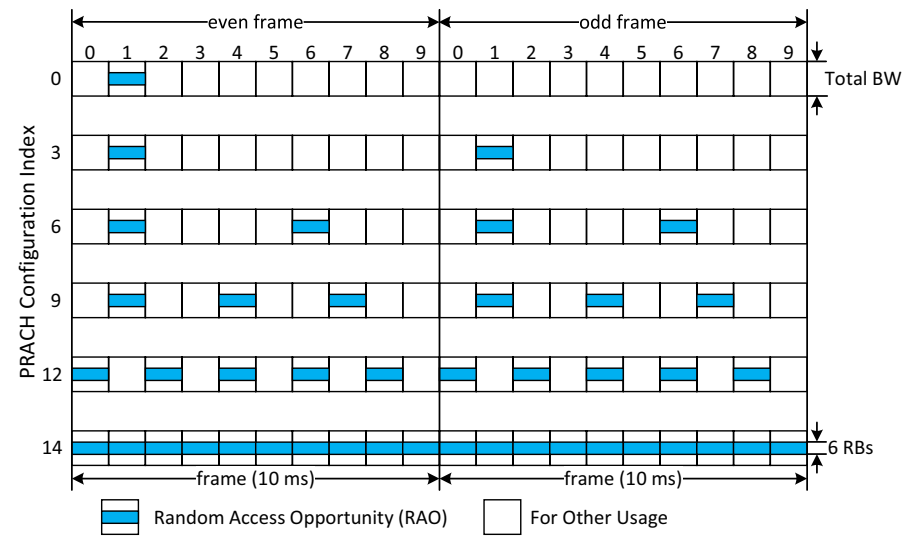

Fig. 2 Example of prach-ConfigIndex

transmission but the uplink synchronization status of the UE shows that it is asynchronous; (vi) handover.

Figure 3 shows the procedure of the existing contention-based 4-step RAP. It can be seen from Fig. 3 that if a UE wants to perform RAP, it must exchange four messages, Msg 1, Msg 2, Msg 3, and Msg 4, with the base station. In general, these messages are also referred to as preamble, random access response (RAR), Radio Resource Control (RRC) connection request, and contention resolution, respectively.

\subsection{Step 1: Msg 1}

Msg 1 is transmitted on the PRACH. As depicted in Fig. 4, the preamble comprises three parts: Cyclic Prefix (CP), Sequence, and Guard time (GT). As listed in Table 3, there are four preamble formats that UE can select. In this table, $T_{\mathrm{CP}}$ is the time duration of the CP and $T_{\mathrm{SEQ}}$ is the time duration of the Sequence. According to the logic root sequence number $u$ in the received SIB2 broadcast by the base station, a UE uses Table 1 to find the corresponding physical root sequence number $u$ and substitutes it into the Zadoff-Chu formula [3], as given in (1), to generate the preamble:

Fig. 3 Contention-based 4-step RAP

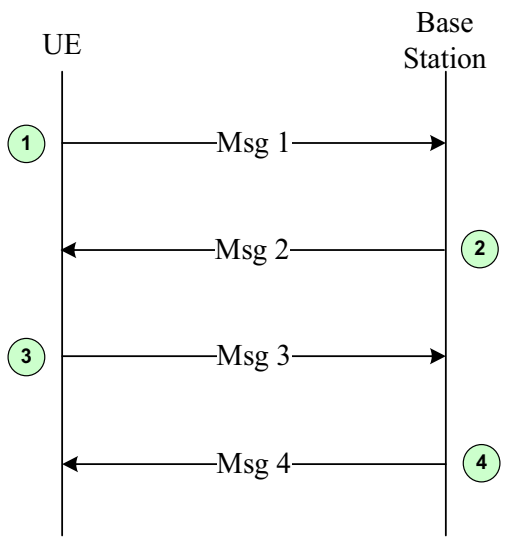


Fig. 4 Preamble format

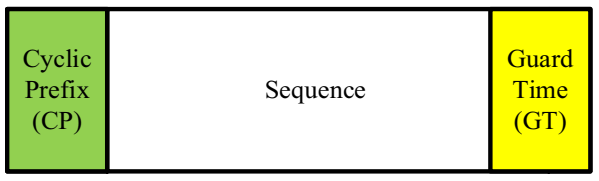

Table 3 Random access preamble parameters [3]

\begin{tabular}{lll}
\hline Preamble format & $T_{\mathrm{CP}}$ & $T_{\mathrm{SEQ}}$ \\
\hline 0 & $3168 \cdot T_{s}$ & $24,576 \cdot T_{s}$ \\
1 & $21,024 \cdot T_{s}$ & $24,576 \cdot T_{s}$ \\
2 & $6240 \cdot T_{s}$ & $2 \cdot 24,576 \cdot T_{s}$ \\
3 & $21,024 \cdot T_{s}$ & $2 \cdot 24,576 \cdot T_{s}$ \\
\hline
\end{tabular}

$$
x_{u}(n)=e^{-j \frac{\pi u n(n+1)}{N_{Z C}}}, \quad 0 \leq n \leq N_{Z C}-1,
$$

where $N_{Z C}=839$ is the number of subcarriers over which UE transmits preamble on the PRACH. The preamble generated from (1) is called the base sequence. By cyclically shifting the base sequence, a UE obtains the complete 64 orthogonal preambles [3]. However, since the base station typically reserves 10 preambles for UEs that perform non-contention-based RAP, the UEs performing contention-based RAP select a preamble randomly from the remaining 54 preambles and uses it as the Msg 1.

Based on the time domain and frequency domain position in which a preamble can be transmitted, a UE forms the Random Access Radio Network Temporary Identifier (RARNTI) [5] as follows:

$$
\text { RA-RNTI }=1+\mathrm{t} \_ \text {id }+10 \times \mathrm{f} \_ \text {id. }
$$

and uses it to scramble the transmitted preamble. As defined in [5], $\mathrm{t} \_$id is the index of the first subframe of the specified PRACH $\left(0 \leq \mathrm{t} \_\mathrm{id}<10\right)$, and $\mathrm{f} \_\mathrm{id}$ is the index of the specified PRACH within that subframe, in ascending order of frequency domain $\left(0 \leq \mathrm{f} \_\mathrm{id}<6\right)$. In FDD mode, f_id will usually be set to 0 . In that case, there is only one RAO for a UE to transmit the preamble in a subframe time in the FDD mode. As shown in Table 4, compared to the FDD mode, in the TDD mode, uplink transmission can only be performed in certain specific subframes [3]. In order to increase the number of RAOs in the TDD mode, more than one chance of RAO is allowed in a subframe time. To achieve this, we can set $\mathrm{f}_{-}$ id to $0-5$, thereby allowing up to 6 RAOs for RAP in one subframe time in the TDD mode.

The RAO selected by a UE to transmit the preamble is based on the value of PRACH Mask Index. As listed in Table 5, the PRACH Mask Index is set to 0 when contentionbased RAP is used, which means that the base station allows the 10 subframes in a frame to be RAOs. In this case, a UE can randomly select one RAO for RAP. B In contrast, for non-contention-based RAP, a UE transmits a preamble on the RAO specified by the PRACH Mask Index to avoid collisions.

Figure 5 shows an example when prach-ConfigIndex broadcast by SIB2 is 12 and PRACH Mask Index is 0. In Fig. 5, among the 5 RAOs numbered 0, 2, 4, 6, and 8, a UE selects the RAO numbered 4 to transmit the preamble. It can also be inferred that the RA-RNTI value of the UE is $1+4+10 \times 0=5$. 
Table 4 Uplink-downlink configurations in the TDD mode

\begin{tabular}{llllllllllll}
\hline Uplink-downlink & Downlink-to-uplink \\
configuration & switch-point periodicity & \multicolumn{1}{ll}{ Subframe number } \\
\cline { 3 - 14 } & & 0 & 1 & 2 & 3 & 4 & 5 & 6 & 7 & 8 & 9 \\
\hline 0 & $5 \mathrm{~ms}$ & $\mathrm{D}$ & $\mathrm{S}$ & $\mathrm{U}$ & $\mathrm{U}$ & $\mathrm{U}$ & $\mathrm{D}$ & $\mathrm{S}$ & $\mathrm{U}$ & $\mathrm{U}$ & $\mathrm{U}$ \\
1 & $5 \mathrm{~ms}$ & $\mathrm{D}$ & $\mathrm{S}$ & $\mathrm{U}$ & $\mathrm{U}$ & $\mathrm{D}$ & $\mathrm{D}$ & $\mathrm{S}$ & $\mathrm{U}$ & $\mathrm{U}$ & $\mathrm{D}$ \\
2 & $5 \mathrm{~ms}$ & $\mathrm{D}$ & $\mathrm{S}$ & $\mathrm{U}$ & $\mathrm{D}$ & $\mathrm{D}$ & $\mathrm{D}$ & $\mathrm{S}$ & $\mathrm{U}$ & $\mathrm{D}$ & $\mathrm{D}$ \\
3 & $10 \mathrm{~ms}$ & $\mathrm{D}$ & $\mathrm{S}$ & $\mathrm{U}$ & $\mathrm{U}$ & $\mathrm{U}$ & $\mathrm{D}$ & $\mathrm{D}$ & $\mathrm{D}$ & $\mathrm{D}$ & $\mathrm{D}$ \\
4 & $10 \mathrm{~ms}$ & $\mathrm{D}$ & $\mathrm{S}$ & $\mathrm{U}$ & $\mathrm{U}$ & $\mathrm{D}$ & $\mathrm{D}$ & $\mathrm{D}$ & $\mathrm{D}$ & $\mathrm{D}$ & $\mathrm{D}$ \\
5 & $10 \mathrm{~ms}$ & $\mathrm{D}$ & $\mathrm{S}$ & $\mathrm{U}$ & $\mathrm{D}$ & $\mathrm{D}$ & $\mathrm{D}$ & $\mathrm{D}$ & $\mathrm{D}$ & $\mathrm{D}$ & $\mathrm{D}$ \\
6 & $5 \mathrm{~ms}$ & $\mathrm{D}$ & $\mathrm{S}$ & $\mathrm{U}$ & $\mathrm{U}$ & $\mathrm{U}$ & $\mathrm{D}$ & $\mathrm{S}$ & $\mathrm{U}$ & $\mathrm{U}$ & $\mathrm{D}$ \\
\hline
\end{tabular}

Table 5 PRACH Mask Index values

\begin{tabular}{lll}
\hline PRACH Mask Index & Allowed PRACH (FDD) & Allowed PRACH (TDD) \\
\hline 0 & All & All \\
1 & PRACH Resource Index 0 & PRACH Resource Index 0 \\
2 & PRACH Resource Index 1 & PRACH Resource Index 1 \\
3 & PRACH Resource Index 2 & PRACH Resource Index 2 \\
4 & PRACH Resource Index 3 & PRACH Resource Index 3 \\
5 & PRACH Resource Index 4 & PRACH Resource Index 4 \\
6 & PRACH Resource Index 5 & PRACH Resource Index 5 \\
7 & PRACH Resource Index 6 & Reserved \\
8 & PRACH Resource Index 7 & Reserved \\
9 & PRACH Resource Index 8 & Reserved \\
10 & PRACH Resource Index 9 & Reserved \\
11 & Every, in the time domain, even PRACH & Every, in the time domain, even PRACH \\
& opportunity 1st PRACH Resource & opportunity 1st PRACH Resource \\
& Index in subframe & Index in subframe \\
12 & Every, in the time domain, odd PRACH & Every, in the time domain, odd PRACH \\
& opportunity 1st PRACH Resource & opportunity 1st PRACH Resource \\
& Index in subframe & Index in subframe \\
13 & Reserved & 1st PRACH Resource Index in subframe \\
14 & Reserved & 2nd PRACH Resource Index in subframe \\
15 & Reserved & 3rd PRACH Resource Index in subframe \\
\hline
\end{tabular}

Fig. 5 RAOs for prach-ConfigIndex $=12$ and PRACH Mask Index $=0$

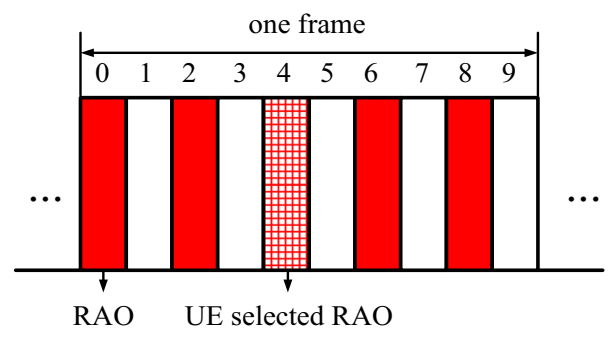




\subsection{Step 2: Msg 2}

The base station uses the Physical Downlink Shared Channel (PDSCH) to transmit Msg 2. Consider the case when multiple UEs transmit randomly selected preambles as their Msg $1 \mathrm{~s}$ in the same RAO. When a preamble is detected at the base station, there might be two possibilities: (i) the detected preamble is transmitted by only one UE; (ii) the detected preamble is transmitted by multiple UEs. Since preambles are orthogonal codes, the base station can only know which preambles are simultaneously transmitted in the same RAO but cannot discern which UEs have transmitted a detected preamble. For each detected preamble, the base station will send a corresponding Msg 2 through the PDSCH. As illustrated in Fig. 6, Msg 2 is divided into MAC header and multiple MAC RARs. Observe that there are many subheaders in the MAC header. Each subheader comprises several fields. ' $E$ ' is Extension and indicates whether there is a MAC subheader in the sequel. ' $\mathrm{T}$ ' is Type and indicates whether what follows is BI or RAPID in the MAC subheader. ' $R$ ' is Reserve and represents reserved bits. Finally, 'BI' is Back Indicator and indicates the required waiting interval before re-sending the preamble in case a UE does not find its corresponding preamble in the received Msg 2. The correspondence between the BI value and the required waiting interval is shown in Table 6 . 'RAPID' stands for Random Access Preamble Identifier and represents the ID of the preamble.

As shown in Fig. 6, if UE wants to receive Msg 2, it must listen to the Msg 2 scrambled by RA-RNTI [1]. After Msg 2 is received, UE first decodes the first subheader in the MAC header to obtain the parameters E, T, and BI. Then, UE decodes each subheader sequentially and determines whether the RAPID in the decoded subheader is the same as the preamble it previously selected in Msg 1 . If they are the same, UE goes to the corresponding MAC RAR to get the RAR information. However, if UE cannot find the same RAPID as was previously sent in Msg 1 in any of the subheaders, it will wait for a backoff time defined by BI as shown in Table 6 before selecting a new preamble and re-sending Msg 1.

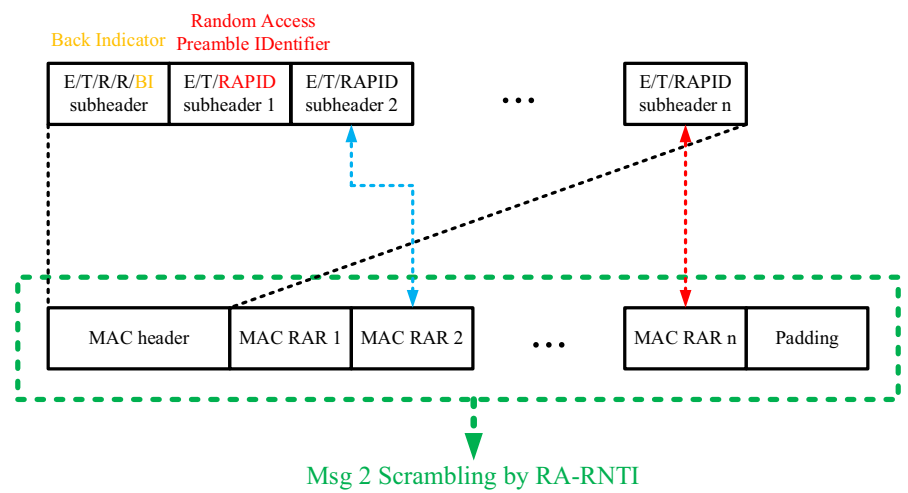

Fig. 6 Illustration of Msg 2 
Table 6 Illustration of back indicator

\begin{tabular}{lccl}
\hline Index & $\begin{array}{l}\text { Backoff Parameter } \\
\text { value (ms) }\end{array}$ & Index & $\begin{array}{l}\text { Backoff } \\
\text { Parameter } \\
\text { value (ms) }\end{array}$ \\
\hline 0 & 0 & 8 & 160 \\
1 & 10 & 9 & 240 \\
2 & 20 & 10 & 320 \\
3 & 30 & 11 & 480 \\
4 & 40 & 12 & 960 \\
5 & 60 & 13 & Reserved \\
6 & 80 & 14 & Reserved \\
7 & 120 & 15 & Reserved \\
\hline
\end{tabular}

\subsection{Step 3: Msg 3}

Msg 3 is transmitted by UE in the Physical Uplink Shared Channel (PUSCH) and is scrambled by Temporary Cell-Radio Network Temporary Identifier (C-RNTI). The time-frequency information of PUSCH is obtained from the MAC RAR of the received Msg 2. The content of Msg 3 includes reasons to establish RRC (3 bits), Type of RRC message (4 bits), UE ID (40 bits), and 1 spare bit, for a total of 48 bits. In [4], the reasons for RRC establishment are emergency, HighPriorityAccess, mt-Access, mo-Signaling, and mo-Data. However, because the reason for RRC establishment is related to the upper layer, it is not clearly stated as to when RRC needs to be established for what reason in [4]. In LTE-A, radio bearers are divided into signaling radio bearer (SRB) for C-plane to carry signaling and data radio bearer (DRB) for U-plane to carry data. The 4-bit Type of RRC message usually uses SRB 0 to carry RRC request signaling. The content of UE ID is based on the RRC connection status. If RAP is performed in the RRC_CONNECTED state, UE ID is the C-RNTI. Otherwise, as in Initial Access, UE ID is a 40-bit random value. In this case, the probability for UEs to have the same UE ID is very small.

Once UE transmits a Msg 3, it starts a timer with a value specified by mac-ContentionResolutionTimer and waits for a Msg 4 from the base station. Since Msg 3 uses the HARQ mechanism, if a Msg 4 is not received before mac-ContentionResolutionTimer expires, UE regards this as contention resolution failure. In this case, if the maximum number of preamble retransmissions is not reached yet, UE waits for a backoff time defined in the received Msg 2 before restarting a new RAP. Conversely, if the maximum number of preamble retransmissions is reached, UE declares a random access failure.

So far, it is known that if multiple UEs select the same preamble and send it to the base station in the same RAO, there may be several UEs that transmit Msg 3 in the allocated PUSCH. These Msg $3 \mathrm{~s}$ sent by different UEs will collide at the base station.

\subsection{Step 4: Msg 4}

The base station uses PDSCH to transmit Msg 4. Msg 4 contains 16 bits of C-RNTI. $\mathrm{C}$-RNTI is the unique radio resource identification code of every UE and is assigned to a UE by the base station. With this identification code, a UE is eligible to use the radio resources of a base station. If a UE does not have a C-RNTI yet, the 40-bit UE ID in Msg 3 
is used as the identification to eliminate Msg 3 contention. When the Msg $3 \mathrm{~s}$ of two UEs collide at the base station, two possible situations may arise: (i) if the base station fails to decode both Msg 3 s, it requests the UEs to retransmit Msg 3; (ii) if the Msg 3 of one UE is successfully decoded, the corresponding UE will get a unique C-RNTI contained in the Msg 4 sent by the base station. The other UE that receives nothing from the base station enters the HARQ procedure for retransmission.

\section{Detailed Design of the Proposed 2-step RAP}

\subsection{2-Step RAP}

Annex B of the 3GPP TR 36.912 [6] provides the signaling exchange structure in each step from C-plane IDLE to CONNECTED and the latency value of the signaling exchange in each step. The document points out that the shortest C-plane latency is $76 \mathrm{~ms}$, and the average latency is $80 \mathrm{~ms}$. The reason for the variation in latency is the unexpected collision of the 4-step RAP. Once a collision occurs, the whole process of the 4-step RAP must be performed again. Consequently, the C-plane latency goes up. Given the severe impact of collision on latency, Ericsson proposed simplifying the 4-step RAP, which is prone to collision, to 2-step RAP to reduce the latency in the C-plane in 2017 [7]. As illustrated in Fig. 7, the Ericsson approach combines Msg 1 and Msg 3 initiated by a UE and Msg 2 and Msg 4 initiated by the base station. Besides, [7] mentions that the 2-step RAP will be mainly applied in the 5G small cell environment, in which the impact of timing alignment (TA) can be ignored. In [7], Ericsson also provides the timing diagrams required for 4-step RAP and 2-step RAP to reduce the C-plane latency to $9 \mathrm{~ms}$.

Since the idea of 2-step RAP was proposed, many proposals were brought up in 3GPP TSG RAN WG1 Meeting \#96 [8-34]. In these proposals, the two messages sent in the 2-step RAP are defined as follows:

(1) Msg A is the message sent in the first step of the 2-step RAP. The combination of Msg 1 and Msg 3 in the 4-step RAP equals Msg A in 2-step RAP.

(2) Msg B is the message sent in the second step of the 2-step RAP. It is the combination of Msg 2 and Msg 4 in the 4-step RAP.

Take the Msg A structure proposed by ZTE [8] as an example. The contents in Msg A are preamble and payload. The preamble is transmitted on PRACH occasion, while the

Fig. 7 2-step RAP [7]

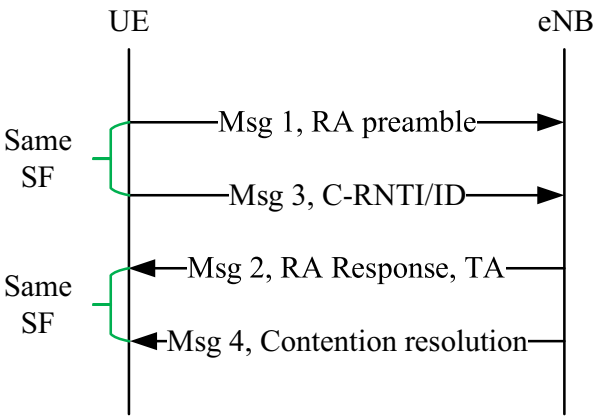


payload is transmitted on the PUSCH occasion. Different from the exact time-domain and frequency-domain information of PUSCH occasion for a UE included in Msg 2 of the 4-step RAP, the uplink radio resources are pre-scheduled by the base station in the 2-step RAP. In the pre-scheduled uplink radio resources, the exact PUSCH occasion for a UE is related to the UE's preamble. Although the basic operation of the 2-step RAP has been defined, many aspects such as what information is to be included in the payload of Msg A, what information is to be sent in Msg B, and which channel is used to transmit Msg B, have not been clearly defined yet.

Due to the limited bandwidth, a UE that does not need to transmit data will be in RRC_ IDLE state. Once a UE wants to transmit data, it must undergo the state transition from RRC_IDLE to RRC_CONNECTED, which is the same for URLLC users. To satisfy the rigorous C-plane latency under the URLLC scenarios, in the proposed design of the 2-step RAP, a UE needs to include a randomly selected UE ID in the payload of Msg A, and the base station should include both C-RNTI and UE ID in Msg B. When a UE transmits the selected preamble in PRACH without collision, there will be no collision when sending the randomly selected UE ID in the payload of Msg A through the corresponding PUSCH. Furthermore, in the proposed design, after receiving Msg A, the base station responds with a Msg B for each decoded preamble in the PDSCH. In each Msg B, a unique C-RNTI and the UE ID in the received Msg A are included. After the completion of the 2-step RAP, the $\mathrm{C}$-RNTI serves as the identifier for a UE to use the radio resources provided by the base station.

\subsection{New SIB2}

In the proposed design of the 2-step RAP, the following three issues are examined: (i) How to enable Msg A to transmit preamble and payload in an RAO? (ii) How to transmit UE ID and DMRS together in the payload? (iii) How to provide a number of dedicated preambles to URLLC users? To address the above three issues, we need to add the following system parameters to the original SIB2:

(1) Newprach-ConfigIndex In the 4-step RAP, RAO only needs to provide the opportunity for a UE to transmit Msg 1 in PRACH. However, RAO in the 2-step RAP offers the UE an opportunity to transmit preamble in PRACH and payload in PUSCH simultaneously. Therefore, newprach-ConfigIndex is introduced to locate the new RAO for the 2-step RAP in the time domain. The detailed description will be given in Sect. 4.3.

(2) $u$ and cyclic shift The base station provides these two parameters for a UE to get the parameters $n_{\mathrm{DMRS}}^{(1)}, n_{\mathrm{DMRS}, \lambda}^{(2)}$, and typeofOCC (orthogonal cover code), needed to generate DMRS. In LTE-A, the cyclic shift is included in the Downlink Channel Information 0 (DCI 0) sent from the base station to the UE through the Physical Downlink Control Channel (PDCCH). For the UE to know these three parameter values before sending Msg A, they must be included in SIB2 and broadcast to the UE. Details will be discussed in Sect. 4.5.

(3) NumberOfURLLC-Preambles The base station uses this parameter to inform the UE about the number of preambles reserved for URLLC users.

(4) Pusch-FreqOffset Since newprach-ConfigIndex has specified the time domain information of the PRACH and PUSCH for sending preamble and payload, the base station uses pusch-FreqOffset to further inform UE about the frequency domain information of the PUSCH. Without loss of generality, this paper assumes it is 0 . 


\subsection{New Random Access Occasion for 2-step RAP}

As shown in Fig. 8, to offer the opportunity for a UE to send Msg A, the new RAO occupies two subframes, one for preamble and one for payload, in the time domain. In the frequency domain, following the 4-step RAP design, the PRACH comprises six consecutive RBs. However, the PUSCH only occupies one RB. Therefore, as shown in Fig. 8, a new RAO defined as the access opportunity occupies two subframes in the time domain and six consecutive RBs for PRACH and one RB for PUSCH in the frequency domain. Since a preamble in the PRACH is mapped to a PUSCH with one RB, the total number of RBs required for the PUSCH is equal to the number of preambles reserved for the URLLC users. For the redesign of RAO, we modify the prach-ConfigIndex in Table 2 to the newprach-ConfigIndex corresponding to the new RAO in the 2-step RAP, as shown in Table 7.

Recall that RA-RNTI is used to scramble Msg 1 in the 4-step RAP. In the 2-step RAP, it is also used to scramble the preamble part of Msg A. However, the payload part of Msg A is scrambled by the RAofPayload-RNTI, which is computed by

$$
\begin{aligned}
R A \text { of Payload-RNTI }= & 1+t \_i d+10 \times f \_i d \\
& +10 \times 6 \times \text { pusch_id },
\end{aligned}
$$

where $t$ id is the index of the first subframe of the specified PRACH $(0 \leq \mathrm{t}$ id $<10)$, f_id is the index of the specified PRACH within that subframe in ascending order in the frequency domain $\left(0 \leq \mathrm{f} \_\mathrm{id}<6\right)$, and pusch_id is the index of the specified PUSCH, in ascending order in the frequency domain $(0 \leq$ pusch_id $<$ numberOfURLLC-Preambles $)$. The maximum value of pusch_id equals the numberOfURLLC-Preambles because the mapping from preamble to $\mathrm{PUSCH}$ occasion is one-to-one in the proposed design, as shown in Fig. 8. Hence, the number of PUSCH occasions equals the number of preambles reserved for the URLLC users.
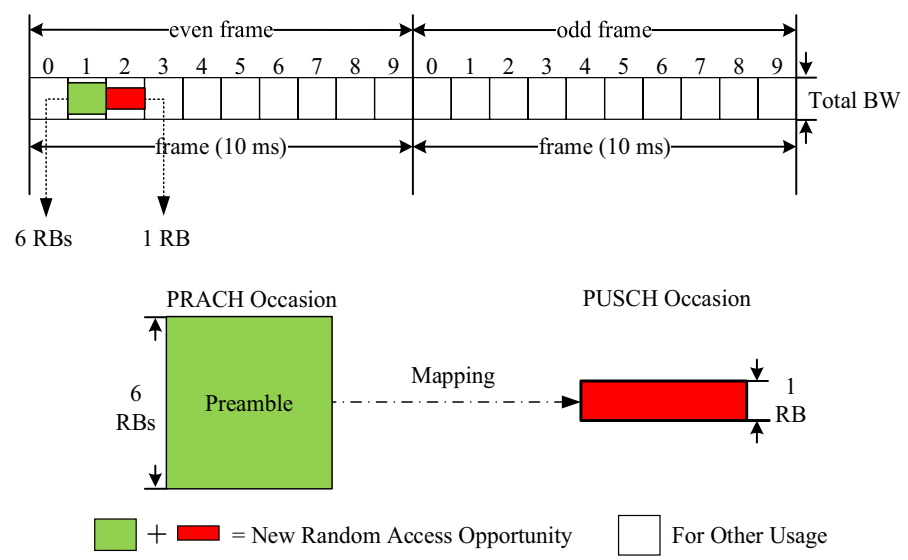

Fig. 8 The mapping of preamble and payload 


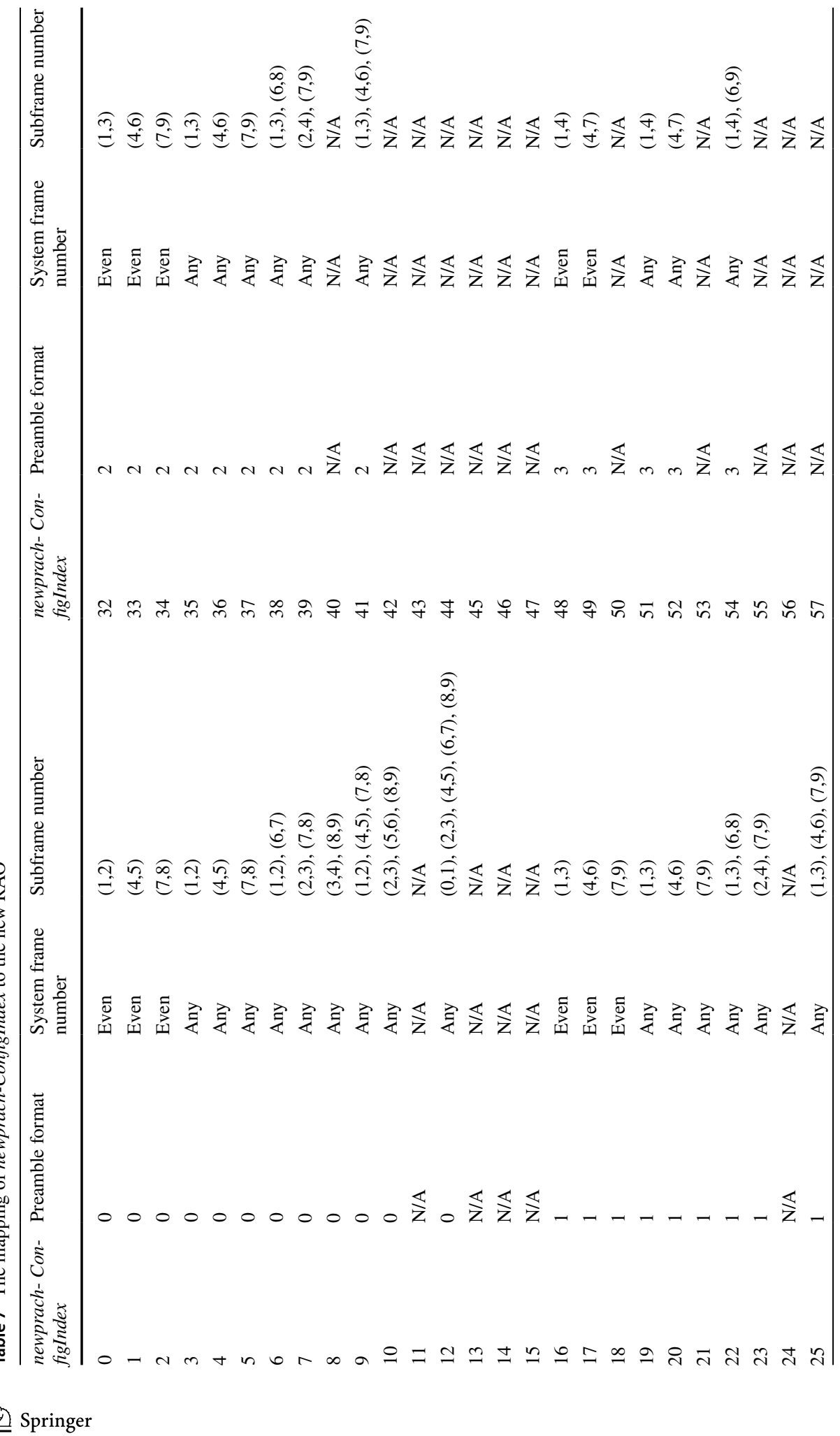




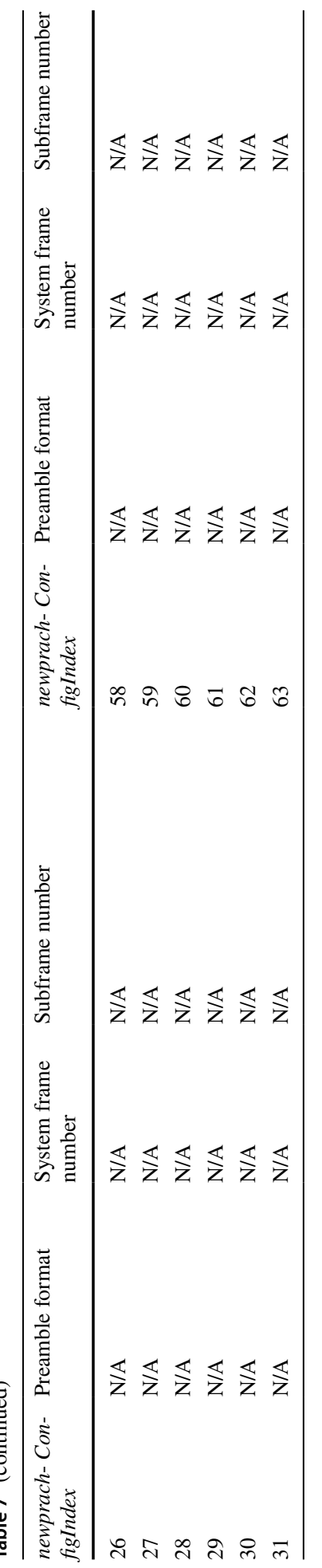




\subsection{The Design of Msg A-Preamble}

As mentioned earlier, for UEs that want to perform contention-based RAP, the preamble in Msg 1 is selected from the 54 preambles reserved by the system. However, if these preambles are not properly allocated to UEs in different application scenarios, it is conceivable that largescale preamble collisions will occur. Therefore, as shown in Fig. 9, the preambles are allocated based on Case 3 of [35] to prevent the preambles selected by the large number of delay-insensitive eMBB users from colliding with those selected by the small number of delay-sensitive URLLC users. In Fig. 9, $m$ is the total number of preambles provided by the base station to the contention-based RAP and $r$ is the number of preambles reserved for URLLC users. It can be seen that although part of the preambles have been reserved for URLLC users, collisions may still occur when several URLLC users select the same preamble.

\subsection{The Design of Msg A-Payload}

Although the 2-step RAP will shorten the time on signaling exchanges, it still suffers the same collision problem as in the 4-step RAP when transmitting the payload. As mentioned in Sect. 4.1, in the design of the 2-step RAP, UE ID needs to be sent in the payload of Msg A. However, in the 2-step RAP, when multiple UEs select the same preamble, each of them will transmit the selected UE ID on the same PUSCH occasion. Thus, collision at the base station is inevitable. In order to reduce the possibility of UE ID collision, the bit string originally used to represent a UE ID in the 4-step RAP is replaced by DMRS signals that exhibit orthogonal characteristics.

In the 4G LTE-A system, when a UE is transmitting uplink data, DMRS is sent in the fourth symbol of a slot for channel estimation. The parameters used by a UE to generate the orthogonal sequence of DMRS are included in DCI 0 initiated by the base station. Since the number of RBs allocated to a UE for the uplink transmission is different, the formulas for generating the DMRS base sequence are also different. In the proposed design, the PUSCH occupies only one RB in the frequency domain. Hence, the formula for generating the DMRS base sequence when only one RB is allocated for a UE to perform uplink transmission is given by

$$
\bar{r}_{u, v}(n)=e^{j \varphi(n) \pi / 4}, 0 \leq n \leq M_{s c}^{R S}-1,
$$

where $M_{\mathrm{sc}}^{\mathrm{RS}}$ is the number of subcarriers used to generate the DMRS base sequence and is set to 12 in this paper. The base sequences are divided into groups, where $u$ is the group number and $v$ is the base sequence number within the group. It is known from Table 8 that there are 30 options for the newly added system parameter $u$ in SIB2. However, since $M_{\mathrm{sc}}^{\mathrm{RS}}$ is set to 12 , there is only one base sequence in each group. Hence, $v$ is set to 0 in this paper.

Case 1

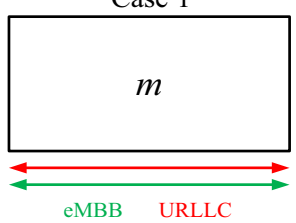

Case 2

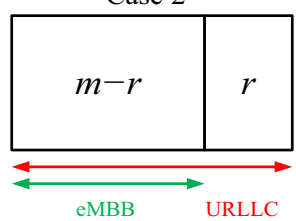

Case 3

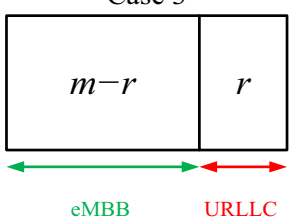

Fig. 9 The allocation of preambles 
Table 8 The value of $u$ [3]

\begin{tabular}{|c|c|c|c|c|c|c|c|c|c|c|c|c|}
\hline \multirow{2}{*}{$\frac{u}{0}$} & \multicolumn{12}{|c|}{$\varphi(0), \ldots, \varphi(11)$} \\
\hline & -1 & 1 & 3 & -3 & 3 & 3 & 1 & 1 & 3 & 1 & -3 & 3 \\
\hline 1 & 1 & 1 & 3 & 3 & 3 & -1 & 1 & -3 & -3 & 1 & -3 & 3 \\
\hline 2 & 1 & 1 & -3 & -3 & -3 & -1 & -3 & -3 & 1 & -3 & 1 & -1 \\
\hline 3 & -1 & 1 & 1 & 1 & 1 & -1 & -3 & -3 & 1 & -3 & 3 & -1 \\
\hline 4 & -1 & 3 & 1 & -1 & 1 & -1 & -3 & -1 & 1 & -1 & 1 & 3 \\
\hline 5 & 1 & -3 & 3 & -1 & -1 & 1 & 1 & -1 & -1 & 3 & -3 & 1 \\
\hline 6 & -1 & 3 & -3 & -3 & -3 & 3 & 1 & -1 & 3 & 3 & -3 & 1 \\
\hline 7 & -3 & -1 & -1 & -1 & 1 & -3 & 3 & -1 & 1 & -3 & 3 & 1 \\
\hline 8 & 1 & -3 & 3 & 1 & -1 & -1 & -1 & 1 & 1 & 3 & -1 & 1 \\
\hline 9 & 1 & -3 & -1 & 3 & 3 & -1 & -3 & 1 & 1 & 1 & 1 & 1 \\
\hline 10 & -1 & 3 & -1 & 1 & 1 & -3 & -3 & -1 & -3 & -3 & 3 & -1 \\
\hline 11 & 3 & 1 & -1 & -1 & 3 & 3 & -3 & 1 & 3 & 1 & 3 & 3 \\
\hline 12 & 1 & -3 & 1 & 1 & -3 & 1 & 1 & 1 & -3 & -3 & -3 & 1 \\
\hline 13 & 3 & 3 & -3 & 3 & -3 & 1 & 1 & 3 & -1 & -3 & 3 & 3 \\
\hline 14 & -3 & 1 & -1 & -3 & -1 & 3 & 1 & 3 & 3 & 3 & -1 & 1 \\
\hline 15 & 3 & -1 & 1 & -3 & -1 & -1 & 1 & 1 & 3 & 1 & -1 & -3 \\
\hline 16 & 1 & 3 & 1 & -1 & 1 & 3 & 3 & 3 & -1 & -1 & 3 & -1 \\
\hline 17 & -3 & 1 & 1 & 3 & -3 & 3 & -3 & -3 & 3 & 1 & 3 & -1 \\
\hline 18 & -3 & 3 & 1 & 1 & -3 & 1 & -3 & -3 & -1 & -1 & 1 & -3 \\
\hline 19 & -1 & 3 & 1 & 3 & 1 & -1 & -1 & 3 & -3 & -1 & -3 & -1 \\
\hline 20 & -1 & -3 & 1 & 1 & 1 & 1 & 3 & 1 & -1 & 1 & -3 & -1 \\
\hline 21 & -1 & 3 & -1 & 1 & -3 & -3 & -3 & -3 & -3 & 1 & -1 & -3 \\
\hline 22 & 1 & 1 & -3 & -3 & -3 & -3 & -1 & 3 & -3 & 1 & -3 & 3 \\
\hline 23 & 1 & 1 & -1 & -3 & -1 & -3 & 1 & -1 & 1 & 3 & -1 & 1 \\
\hline 24 & 1 & 1 & 3 & 1 & 3 & 3 & -1 & 1 & -1 & -3 & -3 & 1 \\
\hline 25 & 1 & -3 & 3 & 3 & 1 & 3 & 3 & 1 & -3 & -1 & -1 & 3 \\
\hline 26 & 1 & 3 & -3 & -3 & 3 & -3 & 1 & -1 & -1 & 3 & -1 & -3 \\
\hline 27 & -3 & -1 & -3 & -1 & -3 & 3 & 1 & -1 & 1 & 3 & -3 & -3 \\
\hline 28 & -1 & 3 & -3 & 3 & -1 & 3 & 3 & -3 & 3 & 3 & -1 & -1 \\
\hline 29 & 3 & -3 & -3 & -1 & -1 & -3 & -1 & 3 & -3 & 3 & 1 & -1 \\
\hline
\end{tabular}

For each value of $u, \varphi(0)$ through (11) constitute the DMRS base sequence and are sent on the fourth symbol of the 12 subcarriers allocated to a UE.

By applying (5) to the DMRS base sequence generated through (4), other DMRS patterns that are orthogonal to the DMRS base sequence are generated.

$$
\bar{r}_{u, v}^{(\alpha)}(n)=e^{j \alpha n} \bar{r}_{u, v}(n), 0 \leq n<M_{\mathrm{sc}}^{\mathrm{RS}},
$$

where the value of $\alpha$ can be obtained by

$$
\begin{gathered}
\alpha=2 \pi n_{c s} / 12, \\
n_{c s}=\left(n_{\mathrm{DMRS}}^{(1)}+n_{\mathrm{DMRS}}^{(2)}+n_{\mathrm{PN}}\left(n_{\mathrm{s}}\right)\right) \bmod 12,
\end{gathered}
$$


Table 9 The parameter $n_{\text {DMRS }}^{(1)}[3]$

\begin{tabular}{lc}
\hline Cyclic shift & $n_{\text {DMRS }}^{(1)}$ \\
\hline 0 & 0 \\
1 & 2 \\
2 & 3 \\
3 & 4 \\
4 & 6 \\
5 & 8 \\
6 & 9 \\
7 & 10 \\
\hline
\end{tabular}

Table 10 The parameter $n_{\text {DMRS }}^{(2)}$ [3]

\begin{tabular}{|c|c|c|c|c|c|c|c|c|}
\hline \multirow{2}{*}{$\begin{array}{l}\text { Cyclic Shift Field in uplink- } \\
\text { related DCI format [36] }\end{array}$} & \multicolumn{4}{|c|}{$n_{\mathrm{DMRS}, \lambda}^{(2)}$} & \multicolumn{4}{|c|}{$\left[w^{(\lambda)}(0) w^{(\lambda)}(1)\right]$} \\
\hline & $\lambda=0$ & $\lambda=1$ & $\lambda=2$ & $\lambda=3$ & $\lambda=5$ & $\lambda=6$ & $\lambda=7$ & $\lambda=8$ \\
\hline 000 & 0 & 6 & 3 & 9 & {$\left[\begin{array}{ll}1 & 1\end{array}\right]$} & {$\left[\begin{array}{ll}1 & 1\end{array}\right]$} & {$[1-1]$} & {$[1-1]$} \\
\hline 001 & 6 & 0 & 9 & 3 & {$[1-1]$} & {$[1-1]$} & {$\left[\begin{array}{ll}1 & 1\end{array}\right]$} & {$\left[\begin{array}{ll}1 & 1\end{array}\right]$} \\
\hline 010 & 3 & 9 & 6 & 0 & {$[1-1]$} & {$[1-1]$} & {$\left[\begin{array}{ll}1 & 1\end{array}\right]$} & [1 1 1 $]$ \\
\hline 011 & 4 & 10 & 7 & 1 & {$\left[\begin{array}{ll}1 & 1\end{array}\right]$} & {$\left[\begin{array}{ll}1 & 1\end{array}\right]$} & {$\left[\begin{array}{ll}1 & 1\end{array}\right]$} & [1 1 1 $]$ \\
\hline 100 & 2 & 8 & 5 & 11 & {$\left[\begin{array}{ll}1 & 1\end{array}\right]$} & {$\left[\begin{array}{ll}1 & 1\end{array}\right]$} & {$\left[\begin{array}{ll}1 & 1\end{array}\right]$} & {$\left[\begin{array}{ll}1 & 1\end{array}\right]$} \\
\hline 101 & 8 & 2 & 11 & 5 & {$[1-1]$} & {$[1-1]$} & {$[1-1]$} & {$[1-1]$} \\
\hline 110 & 10 & 4 & 1 & 7 & {$[1-1]$} & {$[1-1]$} & {$[1-1]$} & {$[1-1]$} \\
\hline 111 & 9 & 3 & 0 & 6 & {$\left[\begin{array}{ll}1 & 1\end{array}\right]$} & {$\left[\begin{array}{ll}1 & 1\end{array}\right]$} & {$[1-1]$} & {$[1-1]$} \\
\hline
\end{tabular}

where $n_{s}$ represents the slot number in a frame with a value between 0 and 19 , and $n_{\mathrm{PN}}\left(n_{s}\right)$ is a value between 0 and 255 [3]. As shown in Tables 9 and 10, by extracting the 3-bit cyclic shift included in the new SIB2, the values of $n_{\mathrm{DMRS}}^{(1)}$ and $n_{\mathrm{DMRS}}^{(2)}$ in (7) can also be obtained. According to (6) and (7), $\alpha$ can produce up to 12 different values, which means that a DMRS base sequence can produce 12 DMRS patterns. Furthermore, by covering (or multiplying) the two forms of typeofOCC, i.e., $\left[w^{(\lambda)}(0) w^{(\lambda)}(1)\right]$, to the 12 DMRS patterns, a total of 24 different DMRS patterns can be obtained. In Table 10, $\lambda$ is the number of transmission layers.

In the 4-step RAP, to reduce the possibility for a large number of UEs to select the same ID, the length of UE ID is set to 40 bits. However, when 5G small cells are considered, the number of URLLC users served by a single $5 \mathrm{G}$ small cell base station will be small. Hence, in the proposed 2-step RAP design, the length of UE ID transmitted in the payload of Msg A is 16 bits. Because different URLLC users may select the same preamble when performing the 2-step RAP, collisions of different payloads transmitted on the same PUSCH may occur. In order to avoid collisions caused by directly transmitting the 16-bit UE ID on the same PUSCH, the 16-bit UE ID is segmented into 4 groups of 4 bits each. Then, instead of sending each group of 4 bits, the DMRS pattern corresponding to a group of 4 bits is sent. For this purpose, 16 out of the 24 DMRS patterns are selected to represent a group of 4 bits. The mapping between a group of 4 bits and 16 DMRS patterns is shown in Table 11. 
Table 11 The mapping between a group of 4 bits and 16 DMRS patterns

\begin{tabular}{|c|c|c|c|c|c|c|c|}
\hline$n_{c s}$ & OCC & DMRS No & 4 bits & $n_{c s}$ & OCC & DMRS No & 4 bits \\
\hline 0 & {$\left[\begin{array}{ll}1 & 1\end{array}\right]$} & 0 & 0000 & 4 & {$\left[\begin{array}{ll}1 & 1\end{array}\right]$} & 8 & 1000 \\
\hline 0 & {$[1-1]$} & 1 & 0001 & 4 & {$[1-1]$} & 9 & 1001 \\
\hline 1 & {$\left[\begin{array}{ll}1 & 1\end{array}\right]$} & 2 & 0010 & 5 & {$\left[\begin{array}{ll}1 & 1\end{array}\right]$} & 10 & 1010 \\
\hline 1 & {$[1-1]$} & 3 & 0011 & 5 & {$[1-1]$} & 11 & 1011 \\
\hline 2 & {$\left[\begin{array}{ll}1 & 1\end{array}\right]$} & 4 & 0100 & 6 & {$\left[\begin{array}{ll}1 & 1\end{array}\right]$} & 12 & 1100 \\
\hline 2 & {$[1-1]$} & 5 & 0101 & 6 & {$[1-1]$} & 13 & 1101 \\
\hline 3 & {$\left[\begin{array}{ll}1 & 1\end{array}\right]$} & 6 & 0110 & 7 & {$\left[\begin{array}{ll}1 & 1\end{array}\right]$} & 14 & 1110 \\
\hline 3 & {$[1-1]$} & 7 & 0111 & 7 & {$[1-1]$} & 15 & 1111 \\
\hline
\end{tabular}

In this way, the transmission of the 16-bit UE ID is replaced by 4 DMRS patterns. Since OCC is employed, when a DMRS pattern is transmitted, the orthogonal sequence must be transmitted in the same symbol of the first and second slots of a subframe. The relationship between the two transmitted orthogonal sequences is determined by the value of OCC, [1 1$]$ for non-reversed or [1-1] for reversed. Assuming that the value of OCC is [1 1] for each group of 4 bits, the 4 DMRS patterns representing the UE ID transmitted on the first symbol to the fourth symbol of the two slots are illustrated in Fig. 10. With this approach, we can ensure that each URLLC user can complete the random access procedure by performing the proposed 2-step RAP only once.

\subsection{Msg B}

In the proposed 2-step RAP design, when the base station organizes the content of Msg B for each received preamble, there will be two possible situations: (i) If the preamble is selected by only one UE, the 16-bit UE ID selected by the UE and a 16-bit C-RNTI issued by the base station are included in Msg B. Therefore, the length of Msg B is 32 bits. (ii) If a preamble is selected by multiple UEs, all the UE IDs and C-RNTIs must be included in the Msg B. In this case, the size of Msg B depends on the total number of UE IDs, which varies due to the false positive problem.

\subsection{False Positive Problem}

If a person does not have COVID-19 infection, s/he should theoretically be tested negative. However, if s/he is tested positive, the diagnosis is false positive. In the proposed 2-step RAP design, the UE ID is transmitted to the base station by using DMRS patterns. Due to the orthogonality of DMRS patterns, there is no payload collision. However, the problem of false positive may occur. Therefore, the problem must be tackled. For example, suppose two UEs select the same preamble \#1 and use the DMRS patterns to transmit their randomly selected

Fig. 10 16-bit UE ID

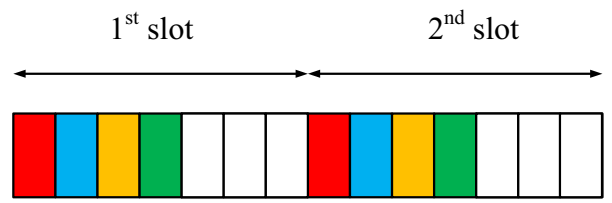


UE IDs on the same payload as shown in Fig. 10. Assume the two randomly selected UE IDs are $(25 \mathrm{C} 9)_{16}$ and $(84 \mathrm{~A} 7)_{16}$ as shown in Table 12. Because of the orthogonality of DMRS patterns, the base station will detect two DMRS patterns in each symbol. After the detection of the four symbols, there will be 16 possible UE IDs as shown in Table 13. However, only 2 of the 16 possible UE IDs are actually transmitted by UEs.

From the above discussion, it is found that when multiple UEs select the same preamble, the false positive problem occurs, which affects the length of Msg B. As shown in Fig. 11, in the proposed 2-step RAP design, only 12 subcarriers are allocated for PDSCH to transmit Msg B. Hence, when the base station needs 4 RBs to transmit Msg B because of the false positive problem, the time-frequency allocation of these $4 \mathrm{RBs}$ is shown in Fig. 11. Therefore, the false positive problem causes variation in the time required by the base station to transmit Msg B.

Consider the case when preamble \#i is detected by the base station. Suppose the numbers of DMRS patterns detected in the first, second, third, and fourth symbols are $n_{1, i}, n_{2, i}, n_{3, i}$, and $n_{4, i}$, respectively. Then, for the detected preamble $\# i,(8)$ is used to calculate the total number of possible UE IDs corresponding to the detected DMRS patterns, (9) is used to determine the total number of UEs selecting the same preamble \# $i$,

Table 12 Illustration of the false positive problem

\begin{tabular}{llllll}
\hline UE No & Preamble No & \multicolumn{2}{l}{ UE ID } & & \\
\hline 1 & $\# 1$ & 2 & 5 & C & 9 \\
2 & $\# 1$ & 8 & 4 & A & 7 \\
Detected at base station & $(2,8)$ & $(5,4)$ & $(\mathrm{C}, \mathrm{A})$ & $(9,7)$ \\
\hline
\end{tabular}

\begin{tabular}{llll}
\hline Combination & UE ID & Combination & UE ID \\
\hline 1 & $(25 \mathrm{C} 9)_{16}$ & 9 & $(85 \mathrm{C} 9)_{16}$ \\
2 & $(25 \mathrm{C} 7)_{16}$ & 10 & $(85 \mathrm{C} 7)_{16}$ \\
3 & $(25 \mathrm{~A} 9)_{16}$ & 11 & $(85 \mathrm{~A} 9)_{16}$ \\
4 & $(25 \mathrm{~A} 7)_{16}$ & 12 & $(85 \mathrm{~A} 7)_{16}$ \\
5 & $(24 \mathrm{C} 9)_{16}$ & 13 & $(84 \mathrm{C} 9)_{16}$ \\
6 & $(24 \mathrm{C} 7)_{16}$ & 14 & $(84 \mathrm{C} 7)_{16}$ \\
7 & $(24 \mathrm{~A} 9)_{16}$ & 15 & $(84 \mathrm{~A} 9)_{16}$ \\
8 & $(24 \mathrm{~A} 7)_{16}$ & 16 & $(84 \mathrm{~A} 7)_{16}$ \\
\hline
\end{tabular}

Fig. 11 Illustration of the timefrequency allocation of Msg B

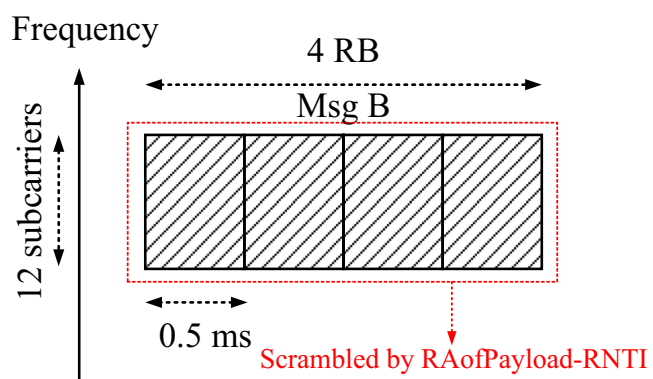

Time 
(10) is used to calculate the number of wasted RBs due to the transmission of a larger Msg B caused by the false positive problem, and (11) is the time wasted in sending the RBs obtained in (10), respectively.

$$
\begin{gathered}
N_{i}=\left(n_{1, i} \times n_{2, i} \times n_{3, i} \times n_{4, i}\right) \\
n_{i}=\max \left(n_{1, i}, n_{2, i}, n_{3, i}, n_{4, i}\right) \\
W_{R B, i}=\left\lceil\left[\left(N_{i}-n_{i}\right) \times 32\right] /(84 \times 6)\right\rceil \\
T_{\text {wasted }, i}=\frac{W_{R B, i}}{2}
\end{gathered}
$$

In 4G LTE-A/5G NR, UE will measure the Signal to Interference plus Noise Ratio (SINR) and then periodically report the channel quality indicator (CQI) to the base station. When a larger CQI value is reported by UE, it means that the downlink channel is in good condition. Therefore, the base station can select a higher modulation order and a larger coding rate. Consequently, the downlink data transmission efficiency is increased. Since $5 \mathrm{G}$ small cells are considered, the modulation order used by the base station in (10) is assumed to be 64 QAM. Hence, each symbol can carry 6 bits. In addition, it is assumed that all the 84 resource elements (REs) in an RB are used to carry the contents of Msg B.

\section{Simulation Results}

\subsection{Simulation Environment}

Two simulation scenarios are considered: fixed number of URLLC users (Scenario 1) and fixed number of preambles reserved for URLLC users (Scenario 2). The latency is defined as the time from a URLLC user starts the RAP to the time it successfully completes the RAP. Let $L_{i}$ be the latency of the $i$-th URLLC user that successfully completes RAP, and $N_{\text {succ }}$ be the number of URLLC users that successfully complete RAP. Among the $N_{\text {succ }}$ URLLC users, $N_{\text {succ }}^{10 \mathrm{~ms}}$ is the number of URLLC users with a latency below $10 \mathrm{~ms}$, and $N_{\text {URLLC }}$ is the total number of URLLC users in the simulation. Three performance metrics are considered in the following. The percentage of RAP successes is defined as

$$
P_{\text {succ }}=\frac{N_{\text {succ }}^{10 \mathrm{~ms}}}{N_{\mathrm{URLLC}}} .
$$

The average latency for the 4-step RAP and 2-step RAP is defined as

$$
L=\frac{1}{N_{\text {succ }}} \sum_{i=1}^{N_{\text {succ }}} L_{i} .
$$

The total number of wasted RBs due to the false positive problem is defined as 


$$
W_{R B, \text { total }}=\sum_{i=1}^{N_{\text {preamble }}} x(i) W_{R B, i} .
$$

where $N_{\text {preamble }}$ is the number of preambles reserved for URLLC users and its value is equal to numberOfURLLC-Preambles. The indicator function $x(i)$ is 1 if preamble \# $i$ is detected by the base station and 0 otherwise. The time for sending the message in each step of the 4-step RAP [6] is listed in Table 14. Note that $T_{\mathrm{Msg} 2}$ includes the processing delay in the base station. Tables 15 and 16 are the parameter values for the 4-step RAP and 2-step RAP in the two considered scenarios, respectively.

Recall that mac-ContentionResolutionTimer is the time that a URLLC user must wait after a collision in Msg 3 before it can start a new round of the 4-step RAP. In Table 15, its value is set to $8 \mathrm{~ms}$. Thus, when a Msg 3 collision occurs, the URLLC users involved must wait for $8 \mathrm{~ms}$ before restarting a new round of the 4-step RAP. However, when a URLLC user fails to receive Msg 2 or Msg 4, a new round of the 4-step RAP will be initiated immediately. Because the parameter value of preambleTransMax is 5, any URLLC user can try the 4-step RAP up to 5 times. In addition, in order to reduce the latency for URLLC users to wait for RAO, the value of prach-ConfigIndex in Table 15 is set to 14 so that each subframe is RAO. Therefore, in the event of a Msg 3 collision, the URLLC user can retransmit Msg 1 in the 4-step RAP at the next RAO after waiting for a time specified by mac-ContentionResolutionTimer. The numberOfURLLC-Preambles is the number of preambles reserved for URLLC users. Based on Table 7, the value of newprach-ConfigIndex in Table 16 is set to 12 so that RAOs can be continuously provided to URLLC users for the

Table 14 The time required to send the message in each step of the 4-step RAP

Table 15 Parameter values for the 4-step RAP

\begin{tabular}{lc}
\hline Parameter & Value \\
\hline Msg 1 transmission time $\left(T_{\mathrm{Msg} 1}\right)$ & $1 \mathrm{~ms}$ \\
Msg 2 transmission time $\left(T_{\mathrm{Msg} 2}\right)$ & $3 \mathrm{~ms}$ \\
Msg 3 transmission time $\left(T_{\mathrm{Msg} 3}\right)$ & $1 \mathrm{~ms}$ \\
Msg 4 transmission time $\left(T_{\mathrm{Msg} 4}\right)$ & $1 \mathrm{~ms}$ \\
\hline
\end{tabular}

\begin{tabular}{lll}
\hline Parameter & Scenario 1 & Scenario 2 \\
\hline Number of URLLC users $\left(N_{\text {URLLC }}\right)$ & 10 & $5,10,15,20$ \\
numberOfURLLC-Preambles & $5,10,15,20$ & 10 \\
mac-ContentionResolutionTimer & $8 \mathrm{~ms}$ & $8 \mathrm{~ms}$ \\
preambleTransMax & 5 & 5 \\
prach-ConfigIndex & 14 & 14 \\
\hline
\end{tabular}

Table 16 Parameter values for the 2-step RAP

\begin{tabular}{lll}
\hline Parameter & Scenario 1 & Scenario 2 \\
\hline Number of URLLC users $\left(N_{\mathrm{URLLC}}\right)$ & 10 & $5,10,15,20$ \\
numberOfURLLC-Preambles & $5,10,15,20$ & 10 \\
newprach-ConfigIndex & 12 & 12 \\
Msg A transmission time $\left(T_{\mathrm{MsgA}}\right)$ & $2 \mathrm{~ms}$ & $2 \mathrm{~ms}$ \\
The number of RBs for PUSCH & $5,10,15,20$ & 10 \\
\hline
\end{tabular}




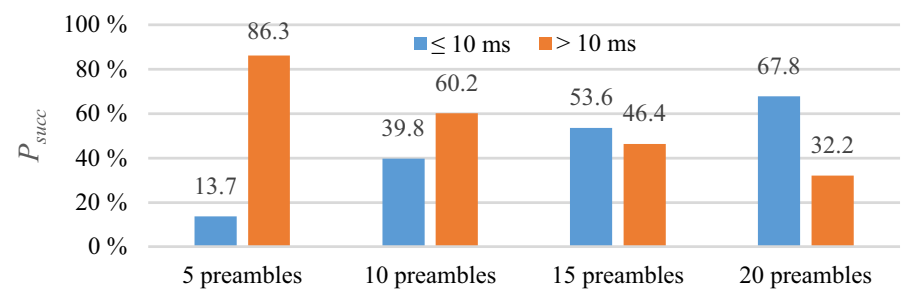

Fig. 12 Percentage of RAP successes of the 4-step RAP with $N_{\text {URLLC }}=10$

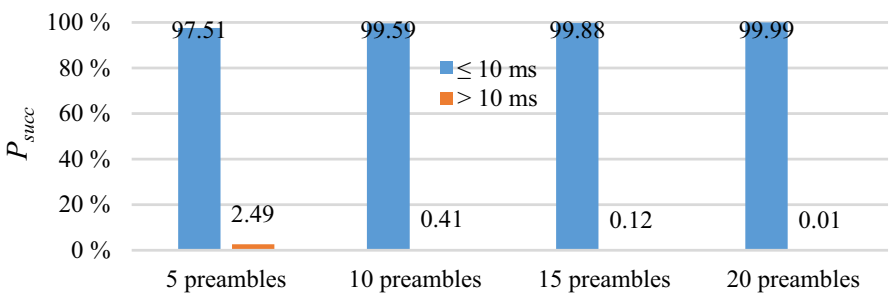

Fig. 13 Percentage of RAP successes of the 2-step RAP with $N_{\text {URLLC }}=10$

2-step RAP. Because preamble and PUSCH are one-to-one mapping as shown in Fig. 8, the total number of RBs allocated for PUSCH is equal to the number of preambles reserved for the URLLC users in the proposed 2-step RAP. Therefore, in Table 16, the number of RBs for PUSCH is equal to the value of the numberOfURLLC-Preambles. The parameter $T_{\mathrm{MsgA}}$ is the time required to send Msg A in the 2-step RAP and is set to 2 ms. However, in the proposed 2-step RAP, the time required to send Msg B varies with the size of Msg B due to the false positive problem.

In this paper, in order to focus on the latency encountered by URLLC users during RAP, both processing delay and latency due to decoding failures are ignored for both 4-step RAP and 2-step RAP. Besides, for the 2-step RAP, the number of wasted RBs due to the false positive problem is calculated regardless of whether URLLC users complete the RAP within $10 \mathrm{~ms}$.

\subsection{Comparison of the Percentage of RAP Successes}

As mac-ContentionResolutionTimer is set to $8 \mathrm{~ms}$, for URLLC users to successfully complete RAP within $10 \mathrm{~ms}$ in the 4-step RAP, there must be no preamble collision in the first round of the 4-step RAP. To achieve this, a preamble can only be selected by one URLLC user. Conversely, when a URLLC user performs the 2-step RAP, as long as the total transmission time of Msg A and Msg B does not exceed $10 \mathrm{~ms}$, the URLLC user is deemed to complete the RAP successfully.

Using the parameter values of Scenario 1 in Tables 15 and 16, 1000 4-step RAP and 1000 2-step RAP simulations are performed, respectively. Based on (12), Figs. 12 and 13 show the obtained percentage of RAP successes $\left(P_{\text {succ }}\right)$ with respect to different numbers of preambles. Figure 12 shows that as the number of preambles reserved for URLLC users increases, the number of preamble collisions in the 4-step RAP decreases. Therefore, $P_{\text {succ }}$ of the 4-step 


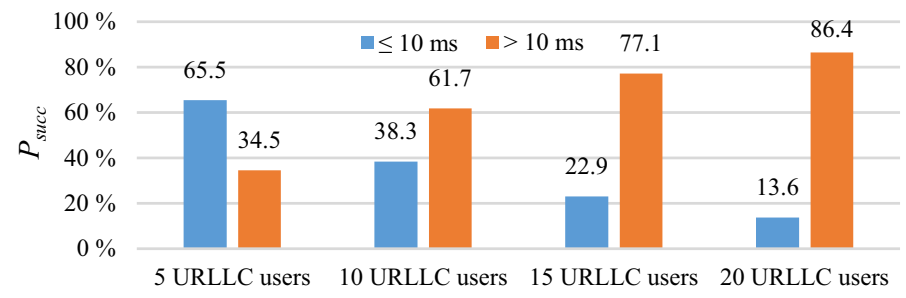

Fig. 14 Percentage of RAP successes of 4-step RAP with numberOfURLLC-Preambles $=10$

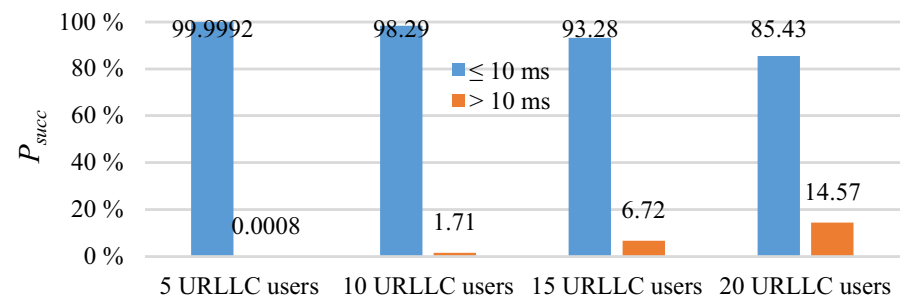

Fig. 15 Percentage of RAP successes of 2-step RAP with numberOfURLLC-Preambles $=10$

RAP will increase accordingly. When 20 preambles are reserved for URLLC users, $P_{\text {succ }}$ of the 4-step RAP reaches the highest 67.8\%. However, because DMRS is introduced in Msg A of the 2-step RAP to transmit UE ID, URLLC users will not re-execute the 2-step RAP when UE ID collision occurs. Therefore, it can be seen from Fig. 13 that $P_{\text {succ }}$ of the 2-step RAP is better than the 4-step RAP. The $P_{\text {succ }}$ gap between these two RAPs is as high as $83.81 \%$.

Figures 14 and 15 show the $P_{\text {succ }}$ obtained when 1000 4-step RAP and 1000 2-step RAP simulations are performed using the parameter values of Scenario 2 in Tables 15 and 16. With the increase of URLLC users, it can be seen from Fig. 14 that $P_{\text {succ }}$ of the 4-step RAP decreases. Since DMRS is introduced in Msg A to transmit UE ID, URLLC users will not reexecute the 2-step RAP when UE ID collision occurs. However, with the increase in the number of URLLC users, the time to transmit Msg B in the 2-step RAP will increase due to the false positive problem. As a result, the latency may exceed $10 \mathrm{~ms}$. Therefore, in Fig. 15, $P_{\text {succ }}$ of the 2-step RAP decreases as the number of URLLC users increases. However, comparison of Figs. 14 and 15 reveals that $P_{\text {succ }}$ of the 4-step RAP is still much lower than that of the 2-step RAP and the largest difference of $P_{\text {succ }}$ between these two figures is $71.83 \%$. In addition, comparison of Figs. 13 and 15 shows that $P_{\text {succ }}$ of the 2-step RAP is more sensitive to the change in the number of URLLC users. The main reason is that as the number of URLLC users increases, the number of URLLC users that simultaneously transmit the UE ID in Msg A will also increase. In this case, the base station needs a longer time to send Msg B due to the false positive problem of the 2-step RAP, as can be seen from (8) to (11). Consequently, the possibility of latency greater than $10 \mathrm{~ms}$ also rises.

\subsection{Comparison of Average Latency}

Since the value of preambleTransMax is set to 5 in Table 15, the 4-step RAP can be repeated at most 5 times before it is successfully completed. In other words, as long 


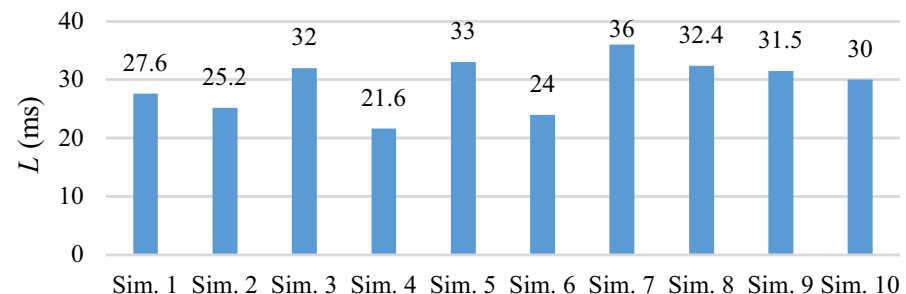

Fig. 16 Average latency of the 4-step RAP with $N_{\mathrm{URLLC}}=10$ and numberOfURLLC-Preambles $=5$

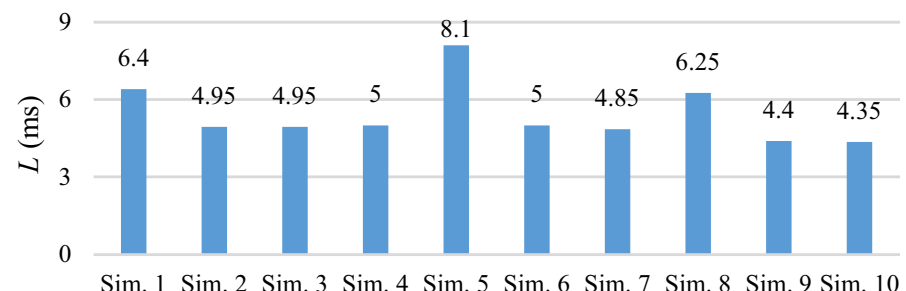

Fig. 17 Average latency of the 2-step RAP with $N_{\mathrm{URLLC}}=10$ and numberOfURLLC-Preambles $=5$

as a URLLC user can successfully complete a 4-step RAP within 5 rounds, the latency incurred by the URLLC user in performing each round of the 4-step RAP is included in the calculation of the average latency. Since there is no preamble collision when using the 2-step RAP, URLLC users always successfully complete the 2-step RAP in one round. However, the time for a URLLC user to successfully complete a 2-step RAP is not fixed. Specifically, the time for a URLLC user to successfully complete a 2-step RAP depends on the outcome of the false positive problem. In the following, two cases with the number of reserved preambles smaller than and greater than the number of URLLC users are considered. Ten simulations are performed for each case.

First, we consider $N_{\mathrm{URLLC}}=10$ and numberOfURLLC-Preambles $=5$ for Scenario 1 in Tables 15 and 16. The average latencies obtained using (13) are shown in Figs. 16 and 17. In these two figures, the maximum average latencies incurred by the 4-step RAP and 2-step RAP are $36 \mathrm{~ms}$ and $8.1 \mathrm{~ms}$, respectively. Similarly, the average latencies for the second case with $N_{\mathrm{URLLC}}=5$ and numberOfURLLC-Preambles $=10$ for Scenario 2 of Tables 15 and 16 are shown in Figs. 18 and 19, respectively. The maximum average latencies incurred by the 4-step RAP and 2-step RAP decrease to $13.2 \mathrm{~ms}$ and $3.5 \mathrm{~ms}$, respectively. The reason is when the number of reserved preambles is smaller than that of URLLC users, the chance of collision of Msg 3 in the 4-step RAP will increase accordingly. Once Msg 3 collision occurs, the average latency will increase too. Therefore, the average latencies in Fig. 16 are higher than those in Fig. 18. Although there is no Msg A collision problem in the 2-step RAP, the length of Msg B in the 2-step RAP increases due to the false positive problem. Besides, from Sect. $4 \mathrm{G}$, the false positive problem exacerbates when the number of reserved preambles is smaller than that of URLLC users. Consequently, longer average latency is observed. Hence, the average latencies in Fig. 17 are higher than those in Fig. 19. Furthermore, once the 4-step RAP encounters Msg 3 collision, it must wait for $8 \mathrm{~ms}$ according to the mac-ContentionResolutionTimer in Table 15 before proceeding to a new round. This is another reason for the 


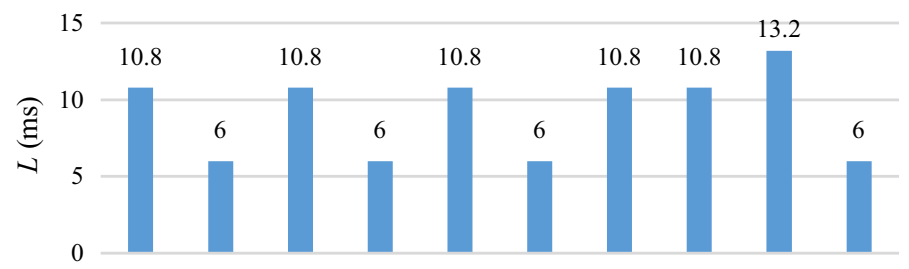

Sim. 1 Sim. 2 Sim. 3 Sim. 4 Sim. 5 Sim. 6 Sim. 7 Sim. 8 Sim. 9 Sim. 10

Fig. 18 Average latency of the 4-step RAP with $N_{\mathrm{URLLC}}=5$ and numberOfURLLC-Preambles $=10$

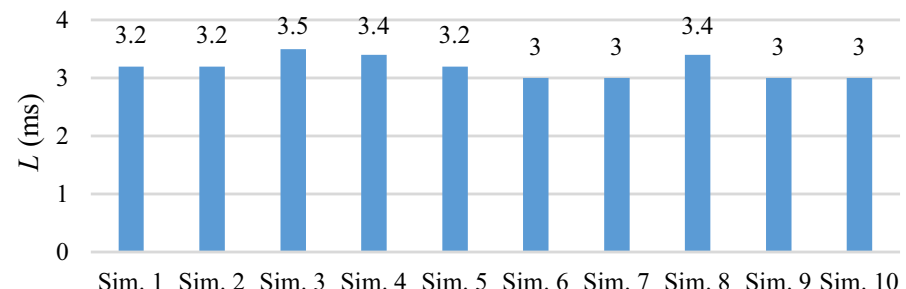

Fig. 19 Average latency of the 2-step RAP with $N_{\mathrm{URLLC}}=5$ and numberOfURLLC-Preambles $=10$

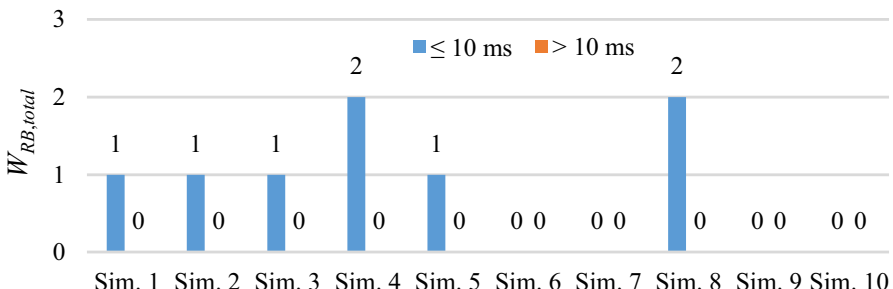

Fig. 20 The total number of wasted RBs with $N_{\mathrm{URLLC}}=5$ and numberOfURLLC-Preambles $=10$

average latency of the 4-step RAP in Figs. 16 and 19 to be higher than that of the 2-step RAP in Figs. 17 and 19, respectively.

\subsection{Total Number of Wasted RBs Due to the False Positive Problem}

For the 2-step RAP, regardless of whether the RAP is completed within $10 \mathrm{~ms}$, as long as multiple URLLC users select the same preamble, a certain number of RBs will be wasted due to the false positive problem. In addition, it can be inferred from (8), (9), and (10) that the number of wasted RBs is proportional to the number of URLLC users that select the same preamble. Based on (14) and the parameter values for Scenario 2 in Table 16, the total number of wasted RBs in each of the 10 simulations for 5, 10, 15, and 20 URLLC users is shown in Figs. 20, 21, 22, and 23, respectively.

First, in Fig. 20, because the number of preambles reserved for the URLLC users is greater than the number of URLLC users, the chance for multiple URLLC users to select the same preamble is low. Compared to the results in Fig. 21, 22, and 23, the total number of wasted RBs in Fig. 20 is the least. Also, a lower number of wasted RBs implies a lower 


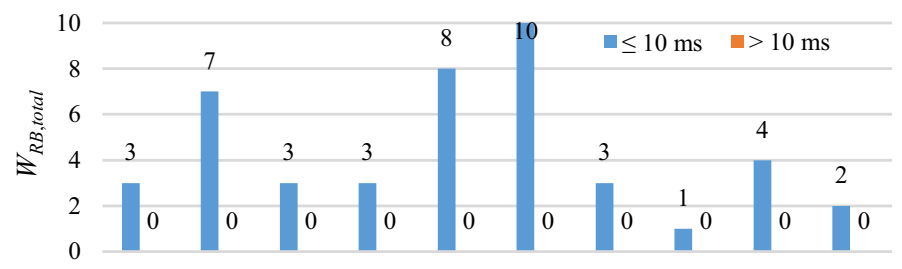

Sim. 1 Sim. 2 Sim. 3 Sim. 4 Sim. 5 Sim. 6 Sim. 7 Sim. 8 Sim. 9 Sim. 10

Fig. 21 The total number of wasted RBs with $N_{\mathrm{URLLC}}=10$ and numberOfURLLC-Preambles $=10$

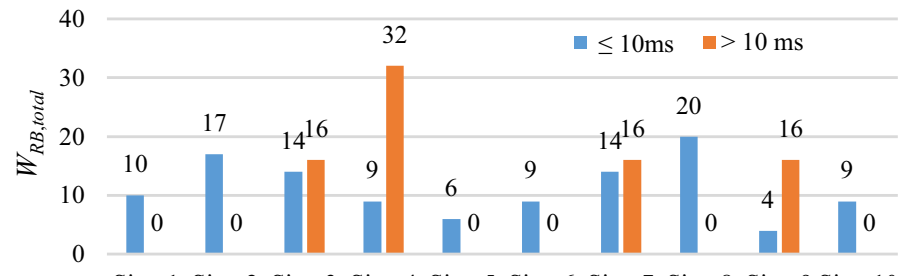

Sim. 1 Sim. 2 Sim. 3 Sim. 4 Sim. 5 Sim. 6 Sim. 7 Sim. 8 Sim. 9 Sim. 10

Fig. 22 The total number of wasted RBs with $N_{\mathrm{URLLC}}=15$ and numberOfURLLC-Preambles $=10$

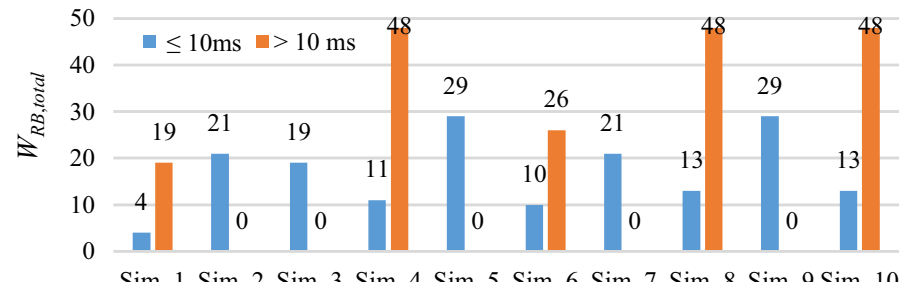

Fig. 23 The total number of wasted RBs with $N_{\mathrm{URLLC}}=20$ and numberOfURLLC-Preambles $=10$

latency for each detected preamble, as shown in (11). As a result, in Fig. 20, all URLLC users can complete the 2-step RAP within $10 \mathrm{~ms}$.

In Fig. 21, the number of URLLC users is equal to the number of preambles reserved for the URLLC users. In this case, the false positive problem grows. Hence, the length of Msg B increases. On the average, the total number of wasted RBs in Fig. 21 is greater than that in Fig. 20. From the simulation results, although the total number of wasted RBs can reach up to 10, all URLLC users can still complete the 2-step RAP within $10 \mathrm{~ms}$.

When the number of URLLC users is greater than the number of preambles reserved for the URLLC users, the possibility for a preamble to be selected by multiple URLLC users increases. Due to the false positive problem, the total number of wasted RBs increases rapidly, as demonstrated in Fig. 22. For the URLLC users with latencies higher than $10 \mathrm{~ms}$, at most 32 wasted RBs are observed in the 4th simulation.

Finally, Fig. 23 demonstrates the total number of wasted RBs when URLLC users are twice that of preambles reserved for URLLC users. Obviously, due to the severe impact of the false positive problem, the total number of wasted RBs increases dramatically. In particular, up to $48 \mathrm{RBs}$ are wasted in the 4 th, 8 th, and 10th simulations. 


\section{Conclusion}

The existing 4-step RAP in 4G LTE-A system cannot meet the C-plane latency requirement of 5G URLLC applications. In view of this, this paper integrates methods such as reserving preamble for URLLC users, simplifying the steps in the 4-step RAP, and using DMRS to represent the UE ID to meet the $10 \mathrm{~ms}$ C-plane latency requirement of 5G. When the number of URLLC users is fixed, the difference in the percentage of URLLC users that complete the 2-step RAP and 4-step RAP within $10 \mathrm{~ms}$ is up to $83.81 \%$. When the number of preambles reserved for URLLC users is fixed, the difference in the percentage of RAP successes between the 2-step RAP and 4-step RAP is up to 71.83\%. Although the proposed 2-step RAP wastes a certain number of RBs to send Msg B due to the false positive problem, this is a minor issue. Therefore, the proposed 2-step RAP is indeed a feasible solution to reduce the C-plane latency.

Funding This paper was supported in part by the Ministry of Science and Technology of Taiwan under the grant number 109-2221-E-197-028-MY3.

\section{Declarations}

Conflict of interest The authors declare that they have no conflict of interests

\section{References}

1. 3GPP (2019). Technical Specification Group Radio Access Network E-UTRA and E-UTRAN Overall Description. TS 36.300, V15.6.0.

2. 3GPP (2018). Study on Scenarios and Requirements for Next Generation Access Technologies. TR 38.913, V15.0.0.

3. 3GPP (2016) Technical Specification Group Radio Access Network E-UTRA Physical channels and modulation. TS 36.211, V13.2.0.

4. 3GPP (2017) Evolved Universal Terrestrial Radio Access (E-UTRA); Radio Control Protocol Specification. TS 36.331. V14.2.0.

5. 3GPP (2017). Technical Specification Group Radio Access Network E-UTRA Medium Access Control (MAC) Protocol Specification. TS 36.321, V13.5.0.

6. 3GPP (2017). Feasibility study for Further Advancements for E-UTRA. TR 36.912, V14.0.0.

7. 3GPP (2017). Text Proposal to TR 38.804 on Random Access Procedure. TSG-RAN WG2 NR Ad Hoc.

8. R1-1901626 (2019). Considerations on The Channel Structure of MsgA. ZTE, 3GPP TSG RAN WG1 Meeting \#96.

9. R1-1901627 (2019). Considerations on 2-Step RACH Procedures. ZTE Sanechips, 3GPP TSG RAN WG1 Meeting \#96.

10. R1-1901669 (2019). Discussion on Channel Structure for 2-Step RACH. vivo, 3GPP TSG RAN WG1 Meeting \#96.

11. R1-1901793 (2019). On Channel Structure for Two-step RACH," MediaTek Inc, 3GPP TSG RAN WG1 Meeting \#96.

12. R1-1902027 (2019). Discussion on Channel Structure for 2-Step RACH," CATT, 3GPP TSG RAN WG1 Meeting \#96.

13. R1-1902133 (2019). Channel Structure for Two-Step RACH Considerations. Sierra Wireless, 3GPP TSG RAN WG1 Meeting \#96. 
14. R1-1902135 (2019). On 2-Step RACH Channel Structure. Nokia, Nokia Shanghai Bell, 3GPP TSG RAN WG1 Meeting \#96.

15. R1-1902165 (2019). On Channel Structure for Two-Step RACH. SONY, 3GPP TSG RAN WG1 Meeting \#96.

16. R1-1902241 (2019). Channel Structure for Two-Step RACH. Samsung, 3GPP TSG RAN WG1 Meeting \#96.

17. R1-1902326 (2019). Discussion on Channel Structure for Two-Step RACH. CMCC, 3GPP TSG RAN WG1 Meeting \#96.

18. R1-1902393 (2019). Discussion on Channel Structure for 2-Step RACH. Panasonic, 3GPP TSG RAN WG1 Meeting \#96.

19. R1-1902466 (2019). Channel Structure for Two-Step RACH. Intel Corporation, 3GPP TSG RAN WG1 Meeting \#96.

20. R1-1902533 (2019). Channel Structure for Two-Step RACH. LG Electronics, 3GPP TSG RAN WG1 Meeting \#96.

21. R1-1902582 (2019). On Signal Structure for Two-Step RACH. InterDigital Inc, 3GPP TSG RAN WG1 Meeting \#96.

22. R1-1902747 (2019). On Channel Structure for 2-Step RACH. OPPO, 3GPP TSG RAN WG1 Meeting \#96.

23. R1-1902784 (2019). Discussion on Channel Structure for Two-Step RACH. NTT DOCOMO, INC, 3GPP TSG RAN WG1 Meeting \#96.

24. R1-1902821 (2019). Use Cases and Scenarios for 2-Step RACH. Ericsson, 3GPP TSG RAN WG1 Meeting \#96.

25. R1-1902822 (2019). Channel Structure for Two-Step RACH. Ericsson, 3GPP TSG RAN WG1 Meeting \#96.

26. R1-1902824 (2019). Evaluation Methodology and Metrics for 2-Step RACH. Ericsson, 3GPP TSG RAN WG1 Meeting \#96.

27. R1-1902917 (2019). Considerations on Resource Pool Design for PUSCH in MsgA of 2-Step RACH. CAICT, 3GPP TSG RAN WG1 Meeting \#96.

28. R1-1902961 (2019). Discussion on Channel Structure for Two-Step RACH. KDDI, 3GPP TSG RAN WG1 Meeting \#96.

29. R1-1902977 (2019). Channel Structure for Two-Step RACH. Qualcomm Incorporated, 3GPP TSG RAN WG1 Meeting \#96.

30. R1-1902979 (2019). Other Considerations for Two-Step RACH," Qualcomm Incorporated, 3GPP TSG RAN WG1 Meeting \#96.

31. R1-1903056 (2019). Channel Structure for 2-Step RACH. Huawei, HiSilicon, 3GPP TSG RAN WG1 Meeting \#96.

32. R1-1903059 (2019). Deployment Scenarios and Design Target for 2-Step RACH. Huawei, HiSilicon, 3GPP TSG RAN WG1 Meeting \#96.

33. R1-1903147 (2019) Considerations on Two-Step RACH for NTN. Thales, 3GPP TSG RAN WG1 Meeting \#96.

34. R1-1903324 (2019) Procedures for Two-Step RACH. Qualcomm Incorporated, 3GPP TSG RAN WG1 Meeting \#96.

35. Chen, Y. J., Cheng, L. Y., \& Wang, L. C. (2017). Prioritized resource reservation for reducing random access delay in 5G URLLC. In 2017 IEEE 28th Annual International Symposium on Personal, Indoor, and Mobile Radio Communications (PIMRC).

36. 3GPP (2019) Eolved Universal Terrestrial Radio Access (E-UTRA); Multiplexing and channel coding. TS 36.212, V16.0.0.

Publisher's Note Springer Nature remains neutral with regard to jurisdictional claims in published maps and institutional affiliations. 


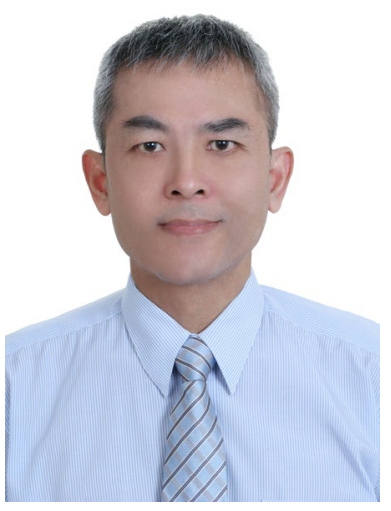

Chih-Cheng Tseng received his B.S. and M.S. from the National Taiwan University of Science and Technology, Taipei, Taiwan, Republic of China, in 1994 and 1997 respectively, all in electronic engineering. He received his $\mathrm{Ph}$. D. from the Graduate Institute of Communications Engineering, National Taiwan University, Taipei, Taiwan, Republic of China, in 2007. He is currently a full professor with the Department of Electrical Engineering, National Ilan University, Yilan, Taiwan, Republic of China. He was a visiting researcher at the Center for TeleInFrastruktur (CTIF), Aalborg University, Denmark on 2007 summer. He has been actively involved in professional activities, serving as a Sponsorship Co-Chair of IEEE Globecom 2020, TPC Co-Chair of Qshine 2015, co-organizer of NGWiFi workshop in IEEE WCNC 2014, HetCarrierCom workshop in IEEE Globecom 2015, 5G CAT workshop in Qshine 2016, and New Radio Technologies workshop in IEEE PIMRC 2017, general secretariat of WPMC 2012, special session organizer of the GWS 2013 and GWS 2014, and reviewer for SCI journals and international conferences. He is currently serving as the editor of the international journals, Physical Communication (SCI), IET Electronic Letters (SCI), and Journal of Mobile Multimedia. He also served as the guest editor of SCI journals such as International Journal of Sensor Networks, Wireless Personal Communications, and Wireless Communications and Mobile Computing. His research interests include the design and performance evaluation of the next generation mobile communication technologies.
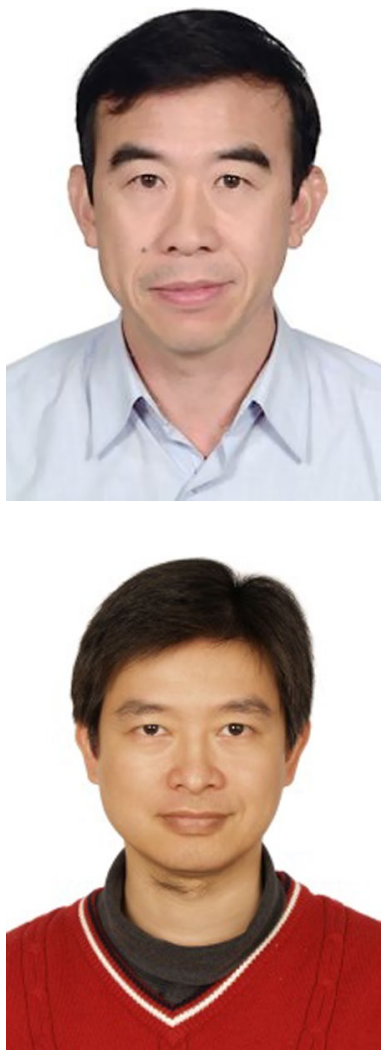

Hwang-Cheng Wang received the M.S. degree in Computer Science and Ph.D. degree in Electrical Engineering, both from the University of Southern California, Los Angeles, California, USA. He is a professor in the Department of Electronic Engineering, National Ilan University (NIU), Taiwan. He is in charge of the Embedded Systems and Mobile Computing Laboratory at NIU. Prior to joining NIU, he was with the Data Communication Institute, Ministry of Transportation and Communications. He has been a visiting scholar to the Next Generation Internet Research Center, Beijing Jiaotong University, Information Science Institute, Academia Sinica, and LNM Institute of Information Technology, India. His research interests and expertise are in mobile communications, embedded systems, internet of things, machine and deep learning, and UAV. He has authored or co-authored journal and conference papers in these areas. He has also served as a technical committee member in international conferences and as a reviewer for reputed journals and conferences.

Jieh-Ren Chang Jieh-Ren Chang received the B.S. and M.S. degrees in nuclear science engineering from National Tsing Hua University, Taiwan, in 1986 and 1988, respectively. He is currently an associate professor in the Department of Electronic Engineering, National Ilan University (NIU), Taiwan. He is in charge of the Intelligent Information Retrival Laboratory at NIU. His major research interests are image processing, soft computing, fuzzy time series, pattern recognition, knowledge discovery from databases and internet web, machine deep learning, big data analysis on industrial manufacture process and innovative combination of information and communication technologies. 

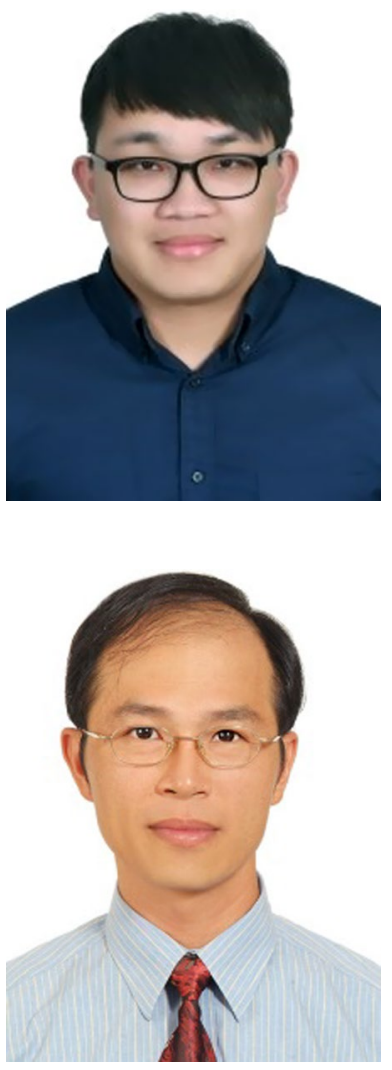

Ling-Han Wang received the MS degree in electrical engineering in 2020 from the National Ilan University, Yilan, Taiwan. He is currently an engineer of the Asia Pacific Telecom Co., Ltd., where he is responsible for the standards of the 3GPP and enterprise $5 \mathrm{G}$ private network. His research interests include wireless communications and $5 \mathrm{G}$ release standards.

Fang-Chang Kuo received the B.S. degree in E.E. from Tatung University in 1986 and Ph.D. degree in E.E. from National Central University, Taiwan, in 1997. He is a professor with the Electronic Engineering department, National Ilan University, Taiwan. He was a visiting scholar with the Department of Computer Science, University of Freiburg, Germany, for one year around 2014. His research interests are in the areas of communication networks and wireless communications. For more information, please refer to kfc.niu.edu.tw. 